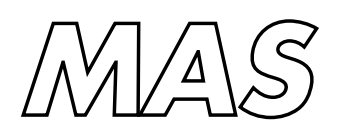

Modelling, Analysis and Simulation

Modelling, Analysis and Simulation
MAS Self-contact for rods on cylinders

G.H.M. van der Heijden, M.A. Peletier, R. Planqué

Report MAS-E0411 August 2004 
CWI is the National Research Institute for Mathematics and Computer Science. It is sponsored by the Netherlands Organization for Scientific Research (NWO).

CWI is a founding member of ERCIM, the European Research Consortium for Informatics and Mathematics.

CWI's research has a theme-oriented structure and is grouped into four clusters. Listed below are the names of the clusters and in parentheses their acronyms.

Probability, Networks and Algorithms (PNA)

Software Engineering (SEN)

Modelling, Analysis and Simulation (MAS)

Information Systems (INS)

Copyright (C) 2004, Stichting Centrum voor Wiskunde en Informatica

P.O. Box 94079, 1090 GB Amsterdam (NL)

Kruislaan 413, 1098 SJ Amsterdam (NL)

Telephone +31205929333

Telefax +31205924199

ISSN 1386-3703 


\title{
Self-contact for rods on cylinders
}

\author{
ABSTRACT \\ We study self-contact phenomena in elastic rods that are constrained to lie on a cylinder. By \\ choosing a particular set of variables to describe the rod centerline the variational setting is \\ made particularly simple: the strain energy is a second-order functional of a single scalar \\ variable, and the self-contact constraint is written as an integral inequality. Using techniques \\ from ode theory (comparison principles) and variational calculus (cut-and-paste arguments) we \\ fully characterize the structure of constrained minimizers. An important auxiliary result states \\ that the set of self-contact points is continuous, a result that contrasts with known examples \\ from contact problems in free rods.
}

2000 Mathematics Subject Classification: 34C11, 34C25, 34C60, 47J30, 49J40, 74G25, 74G55, 74G65

Keywords and Phrases: elastic rods, calculus of variations, constrained minimization, self-contact, comparison principle Note: This research was partly carried out under project MAS 1.1, "PDE's in the Life Sciences" 


\title{
SELF-CONTACT FOR RODS ON CYLINDERS
}

\author{
GERT H. M. VAN DER HEIJDEN†, MARK A. PELETIER $\ddagger$ AND ROBERT PLANQUÉ§
}

\begin{abstract}
We study self-contact phenomena in elastic rods that are constrained to lie on a cylinder. By choosing a particular set of variables to describe the rod centerline the variational setting is made particularly simple: the strain energy is a second-order functional of a single scalar variable, and the self-contact constraint is written as an integral inequality.

Using techniques from ode theory (comparison principles) and variational calculus (cut-and-paste arguments) we fully characterize the structure of constrained minimizers. An important auxiliary result states that the set of selfcontact points is continuous, a result that contrasts with known examples from contact problems in free rods.
\end{abstract}

Key words: elastic rods, calculus of variations, constrained minimization, selfcontact, comparison principle.

2000 Mathematics Subject Classification: 34C11, 34C25, 34C60, 47J30, 49J40, 74G25, 74G55, 74G65.

Note: This research was partly carried out under project MAS 1.1, "PDE's in the Life Sciences".

\section{InTRODUCTION}

The study of self-contact in elastic rods has seen some remarkable progress over the last ten years, with highlights such as the numerical work of Tobias, Coleman, and Swigon $[22,6,5]$, the introduction of global curvature by Gonzalez and co-workers [10], and the derivation of the Euler-Lagrange equations for energy minimization by Schuricht and Von der Mosel [19]. Parallel advances have been made on the highly related ideal knots and Gehring links, where ropelength is minimized instead of elastic energy $[4,18,3]$.

Despite this progress important questions remain open. We are still far from understanding analytically the solutions of the Euler-Lagrange equations for general contact situations. Even if we limit ourselves to global minimizers of an appropriate energy functional, we can prove little about the form of solutions as soon as contact is taken into account.

For instance, a long-standing conjecture for closed elastic rods is that in the limit of long rods under constant twist the global energy minimizer should be a ply (double helix) with a loop on each end. If a structure of this type is assumed, then the limiting pitch angle can be determined [21]; but the difficult part actually consists in showing that global minimizers have this structure. Incidentally, since local minimizers of different type have been found numerically $[6,5]$, the restriction to global minimizers appears to be essential.

Submitted to Archive for Rational Mechanics and Analysis. 
This example is typical for the current state of understanding: if assumptions are made on the set of contacts, then characterizations are possible [8, 20, 21, 12], but for unrestrained geometry little is known rigorously. It shows how our lack of understanding of energy minimizers is intimately linked to the lack of knowledge about structure of the contact set. Examples show that this structure can be nontrivial: for instance, non-contiguous contact appears at the end of a ply in an elastic $\operatorname{rod}[5]$.

In this paper we study a problem of self-contact of an elastic rod in which the rod has reduced freedom of movement: the centerline of the rod is constrained to lie on the surface of a cylinder (Figure 1). In contrast to the full three-dimensional problem referred to above, the reduced dimensionality of this problem enables us to give a near-complete characterization of global minimizers, without making any a priori assumptions on the structure of the contact set. Notwithstanding this, determining the structure of the contact set is a central element of this paper.

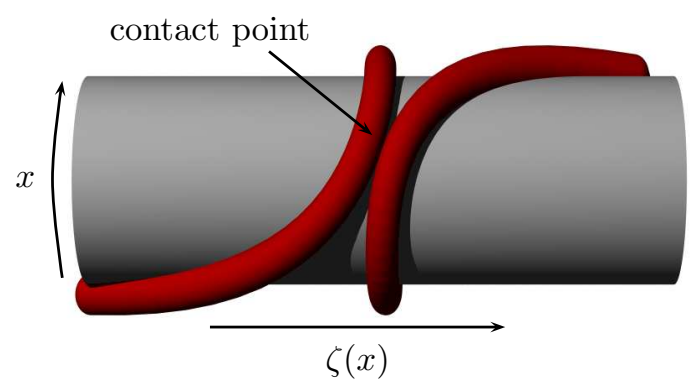

FiguRE 1. The centerline of a rod on a cylinder is described using cylindrical coordinates: the independent variable $x$ is the tangential coordinate, and the position of the centerline is given by the function $\zeta(x)$ measuring distance along the cylinder axis.

We transform the classical Cosserat model of an elastic, unshearable rod of circular cross-section into a more convenient form. The functional that is to be minimized (representing stored energy and work done by the end moment) is

$$
F(u)=\int_{0}^{T}\left[a(u) u^{\prime 2}+b(u)\right]
$$

where

$$
a(u)=\frac{1}{4 \pi^{2}} \frac{1}{\left(1+u^{2}\right)^{5 / 2}} \quad \text { and } \quad b(u)=\frac{1}{r^{2}\left(1+u^{2}\right)^{3 / 2}}-\frac{2 M}{B r} \frac{\sqrt{1+u^{2}}-u}{\sqrt{1+u^{2}}} .
$$

Here $r$ is the radius of the cylinder, $M$ the moment applied to the end of the rod, and $B$ is the bending coefficient of the rod. The centerline of the rod is characterized by $\zeta(x)$, which measures distance along the cylinder axis as a function of a tangential independent variable $x$. The unknown in this minimization problem is the derivative $u(x)=\zeta^{\prime}(x)$, which may by thought of as the cotangent of the angle between the centerline tangent and the cylinder axis; $u$ is zero when the rod curls around the cylinder orthogonal to the axis, and $u= \pm \infty$ when the rod is parallel to the axis. This transformation is detailed in Section 3. 
The most interesting part of the variational problem is the transformed contact condition (condition of non-self-penetration). In this paper we take the thickness of the rod to be zero; then the non-self-penetration condition is

$$
\int_{x}^{x+1} u \geq 0 \quad \text { for all } \quad 0 \leq x \leq T-1
$$

where the interval $[x, x+1]$ corresponds to one full turn around the cylinder; this condition formalizes the intuitive idea that non-self-penetration is equivalent to the condition 'that the rod remain on the same side of itself'. This condition on $u$ makes the variational problem a non-local obstacle problem. Non-zero thickness requires a contact condition that is substantially more involved than (2); we comment on this situation in Section 3.5.

Both the background in rod theory and the independent mathematical context of this minimization problem raise questions about the solutions:

(1) Do solutions exist?

(2) What is the minimal, and what is the maximal regularity of minimizers?

(3) When is there contact, i.e., when is the contact set

$$
\omega_{c}:=\left\{x \in[0, T-1]: \int_{x}^{x+1} u=0\right\}
$$

non-empty?

(4) Given that $\omega_{c} \neq \emptyset$, what is the structure of $\omega_{c}$ ? Is the contact simply contained in a single interval, or is the structure more intricate, as in the examples of contact-skip-contact at the end of a ply [6] and in a (ropelength minimizing) clasp [3]?

(5) What form do the contact forces take?

(6) Does the solution inherit the symmetry of the formulation? This is the case for a symmetric rod on a cylinder without contact condition [11], but need not be true when taking contact into account.

In the rest of this paper we address these questions.

\section{Results}

The first main result of this investigation (Theorem 4.2) shows that the contact condition (2) is essential: without this condition the centerline of a rod will intersect itself. A little experiment with some string wrapped around a pencil will convince the reader that this is the case. We also prove the regularity result that a constrained minimizer $u$ is of class $W^{2, \infty}$, and we derive the Euler-Lagrange equation

$$
N(u)(x):=-2 a(u(x)) u^{\prime \prime}(x)-a^{\prime}(u(x)) u^{\prime 2}(x)+b^{\prime}(u(x))=\int_{x-1}^{x} f,
$$

where the Lagrange multiplier $f$ is a non-negative Radon measure with support contained in the contact set $\omega_{c}$ (Theorem 5.1).

From stationarity alone, which is the basis of Theorem 5.1, the characterization of $f$ as a positive Radon measure appears to be optimal; no further information can be extracted. In Section 7 we use two different additional assumptions to further characterize the contact set and subsequently the measure $f$. In both cases we obtain the important result that the contact set is a (possibly empty) interval and 
that the measure $f$ is a sum of Dirac delta functions, as represented schematically in Figure 2. The weighting of the delta functions is shown in the middle of Figure 2: there is a linear decrease or increase in weight from one side of the contact set to the other (Theorem 6.6). Since $f$ may be interpreted as the contact force, we deduce that

- The contact force is concentrated in at most two tangential positions $x_{1}$ and $x_{2}$, and in integer translates of $x_{1,2}$;

- The magnitude of the contact force is maximal at the contact point where the rod lifts off, and decreases linearly with each turn. Figure 3 graphically illustrates this behaviour.
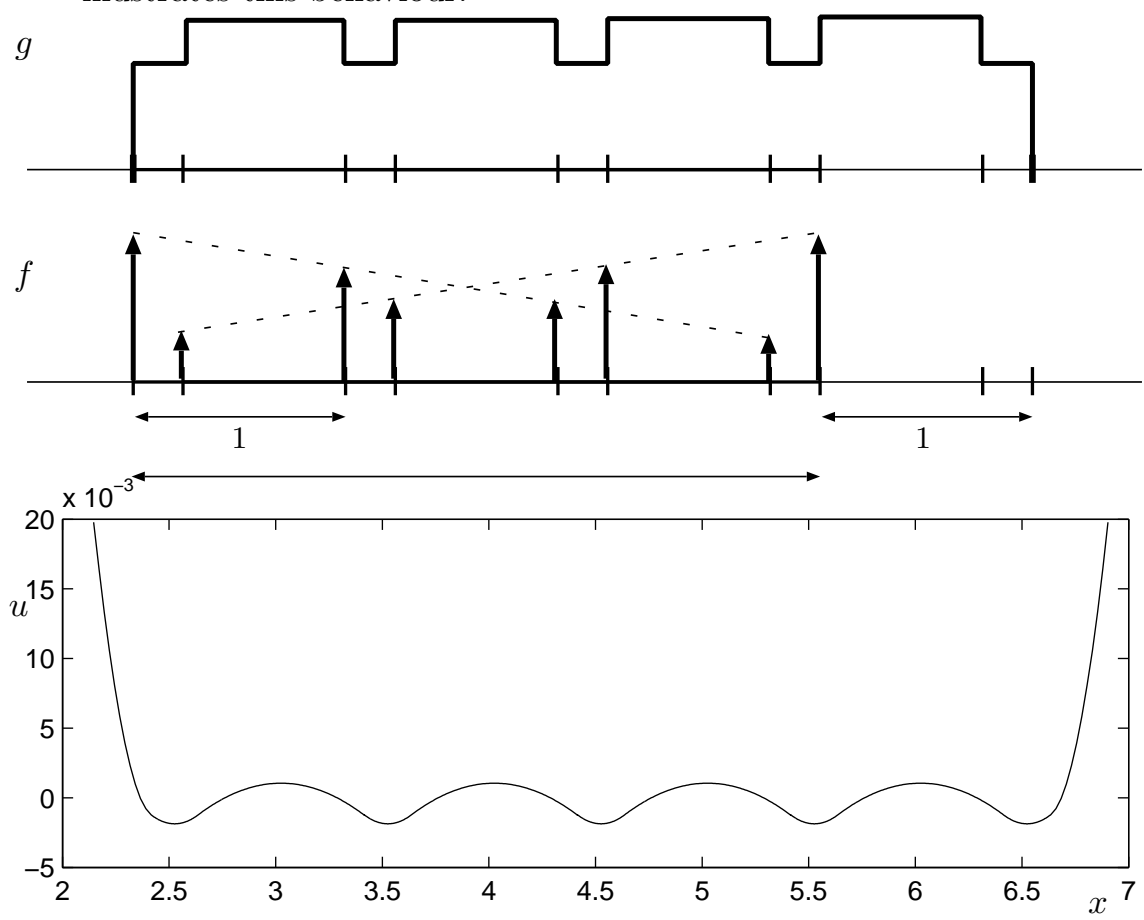

FiguRE 2. The function $g(x)=\int_{x-1}^{x} f$ is piecewise constant (top); the jumps correspond to Dirac delta functions in $f$ (middle). Note that the support of $g$ is the set $\omega_{c}+[0,1]$ by the definition of $g$. The solution $u$ corresponding to $f$ and $g$ is shown at the bottom.

The decrease in contact force with each turn can be understood in the following way. The difference between the contact forces on either side of the rod creates a resulting force exerted on the rod, and the two resultant forces that act at $x_{1,2} \bmod 1$ point in opposite directions. If we imagine a single, closed ring with two forces acting on it in this way, the two forces create a moment that will bend the ring. This also happens with the coil of the current problem, as is demonstrated by the small but definite oscillations in the numerical solutions calculated in Section 9.

As mentioned above, the crucial result that the contact set is connected requires additional assumptions. If we step back from this rod-on-cylinder model, and allow $a$ and $b$ to be general given functions, then for a large class of such functions 

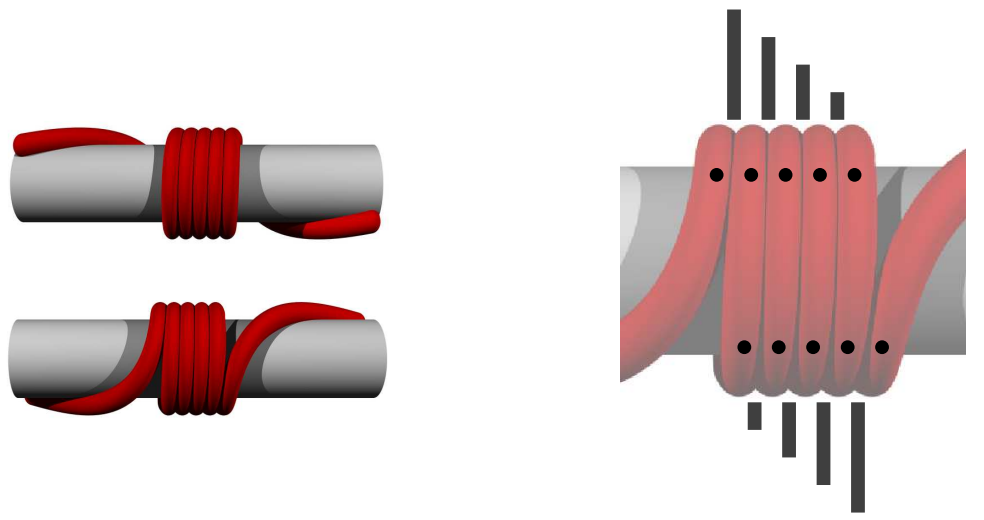

FiguRE 3. A typical rod configuration (left; front and back views) that minimizes energy and satisfies the contact condition. On the right the bars indicate the contact forces corresponding to the arrows in Figure 2. (The analysis of this paper assumes zero rod thickness - in this picture the rod has been fattened for presentation purposes.)

the nonlinear operator on the left-hand side of (4) $N(u)$ satisfies a version of the comparison principle,

$$
N u_{1} \geq N u_{2} \quad \Longrightarrow \quad u_{1} \geq u_{2}
$$

(see Definition 7.1 for the precise statement). For such functions $a$ and $b$, any stationary point has a connected contact set (Theorem 7.3). The argument is based on the observation that non-contact in some interval $(\alpha, \beta)$ implies that $f=0$ on $(\alpha, \beta)$ and therefore that the right-hand side of $(4)$,

$$
g(x)=\int_{x-1}^{x} f,
$$

is non-increasing on $(\alpha, \beta)$ and non-decreasing on $(\alpha-1, \beta-1)$.

Importantly, however, the functions $a$ and $b$ given in (1) are such that the associated operator mostly fails to satisfy this comparison principle. We therefore also take a different approach, in which we obtain the same result by only considering global minimizers. Here we use a different argument, based on constructing other minimizers by cutting and pasting; the combined condition of minimization and non-contact in an interval $(\alpha, \beta)$ implies the existence of additional regions of non-contact outside of the interval $(\alpha, \beta)$, implying that the right-hand side of (4) is constant on $(\alpha, \beta)$. From this the result follows (Theorem 7.4).

In both cases, the fact that the contact set is an interval implies that the boundary of the contact set is 'free' - the measure $f$ is zero on an additional interval of length one extending on both ends of $\omega_{c}$. This implies that the right-hand side $g$ is increasing and decreasing at the same time - except at points that lie at integer distance from the two boundary points. This imposes the specific structure on $g$ and $f$ that is shown in Figure 2 .

The issue of symmetry of minimizers is a subtle one, which again depends on the presence or absence of a comparison principle. The comparison principle simplifies 
the structure of solutions: all stationary points are symmetric (up to an unimportant condition on $b$ ). Without a comparison principle, and more precisely when minimization of $F$ favours oscillation, this is no longer true, and even stationary points that are global minimizers may be asymmetric (Section 8).

Using the characterization of $f$ and $g$ derived earlier we use two numerical methods to investigate constrained minimizers (Section 9); one is a method of direct solution, using a boundary-value solver, and the other a continuation method. A typical solution is shown in Figure 3.

The simple structure of the functional and the contact condition suggest that the methods and results of this paper might be applicable to other systems than this particular rod-on-cylinder model. We therefore state and prove our results for general functions $a$ and $b$. The main requirements are that $a$ and $b$ are smooth and that $a$ is positive; other conditions are mentioned in the text below.

\section{Problem Setting: DeRivation of the ROD-ON-CYLINDER MODEL}

3.1. Kinematics. Consider an elastic rod of circular cross-section that is constrained to lie on a cylinder, and which is subject to a force $T$ and a moment $M$ at the ends. We assume that at the rod ends, $T$, and $M$ are maintained parallel to both the axis of the cylinder and the axis of the rod, but that the loading device leaves the rod ends free to rotate around the circumference of the cylinder; the ends of the rod therefore need not be coaxial. The rod is naturally straight and inextensible, and material cross-sections are assumed to remain orthogonal to the centerline. We will derive a minimization problem for rods of length $2 \ell$ and later take the limit $\ell \rightarrow \infty$.

In the Cosserat rod theory $[1, \mathrm{Ch}$. VIII] the configuration of this rod is characterized by a right-handed orthogonal rod-centered coordinate frame of directors, $\left\{\boldsymbol{d}_{1}, \boldsymbol{d}_{2}, \boldsymbol{d}_{3}\right\}$, each a function of the arc-length parameter $s$. The director $\boldsymbol{d}_{3}$ is assumed parallel to the centerline tangent, and by the assumption of inextensibility the centerline curve $\boldsymbol{r}$ satisfies

$$
\dot{\boldsymbol{r}}=\boldsymbol{d}_{3},
$$

where the dot denotes differentiation with respect to arc length. The strain of the rod is characterized by the vector-valued function $\boldsymbol{u}$ given by

$$
\dot{\boldsymbol{d}}_{k}=\boldsymbol{u} \times \boldsymbol{d}_{k}, \quad k=1,2,3 .
$$

When decomposed as $\boldsymbol{u}=\kappa_{1} \boldsymbol{d}_{1}+\kappa_{2} \boldsymbol{d}_{2}+\tau \boldsymbol{d}_{3}$, the components may be recognized as the two curvatures $\kappa_{1,2}$ and the twist $\tau$.

We choose a fixed frame of reference $\left\{\boldsymbol{e}_{1}, \boldsymbol{e}_{2}, \boldsymbol{e}_{3}\right\}$, where $\boldsymbol{e}_{3}$ is parallel to the cylinder axis, and we relate the frame $\left\{\boldsymbol{d}_{1}, \boldsymbol{d}_{2}, \boldsymbol{d}_{3}\right\}$ to this frame by a particular choice of Euler angles $\{\theta, \psi, \phi\}[14,11]$. In this parametrization $\theta$ is the angle between $\boldsymbol{d}_{3}$ and $\boldsymbol{e}_{3}$ (or between the centerline and the cylinder axis), $\psi$ characterizes the rotation around the cylinder axis, and $\phi$ is a partial measure of the rotation between cross-sections. The condition that the centerline of the rod lie on the surface of a cylinder of radius $r$ translates into the kinematic condition

$$
\dot{\psi}=\frac{1}{r} \sin \theta
$$

where the dot denotes differentiation with respect to the arclength coordinate $s$. Note that it is natural not to restrict $\psi$ to an interval of length $2 \pi$. In terms of the 
remaining degrees of freedom $\{\theta, \phi\}$ the curvatures and twist are given by

$$
\begin{aligned}
\kappa_{1} & =\dot{\theta} \sin \phi-\frac{1}{r} \sin ^{2} \theta \cos \phi, \\
\kappa_{2} & =\dot{\theta} \cos \phi+\frac{1}{r} \sin ^{2} \theta \sin \phi, \\
\tau & =\dot{\phi}+\frac{1}{r} \sin \theta \cos \theta .
\end{aligned}
$$

3.2. Energy, work, and a variational problem. For a given rod the strain energy is given by [14],

$$
\begin{aligned}
E(\theta, \tau) & =\frac{B}{2} \int_{-\ell}^{\ell}\left(\kappa_{1}^{2}+\kappa_{2}^{2}\right)+\frac{C}{2} \int_{-\ell}^{\ell} \tau^{2} \\
& =\frac{B}{2} \int_{-\ell}^{\ell} \dot{\theta}^{2}+\frac{B}{2 r^{2}} \int_{-\ell}^{\ell} \sin ^{4} \theta+\frac{C}{2} \int_{-\ell}^{\ell} \tau^{2} .
\end{aligned}
$$

Here $B$ and $C$ are the bending and torsional stiffnesses respectively. To determine the work done by the tension and moment at the ends of the rod we need to characterize the generalized displacements associated with these generalized forces. For the tension $T$ the associated displacement is the shortening $S$,

$$
S(\theta)=\int_{-\ell}^{\ell}(1-\cos \theta) .
$$

The generalized displacement associated with the moment $M$ is the end rotation, which is well-defined by the assumption of constant end tangents. It is common to identify the end rotation with a link-like functional

$$
L=\int_{-\ell}^{\ell}(\dot{\phi}+\dot{\psi})=[\phi+\psi]_{-\ell}^{\ell} .
$$

As demonstrated in [13], this identification is correct in an open set around the undeformed configuration $\theta \equiv 0$, but loses validity when $|\theta|$ takes values larger than $\pi$. Although nothing we have seen suggests that in an energy-minimizing situation $\theta$ would take values outside of the admissible interval $(-\pi, \pi)$, we have no rigorous argument to guarantee that $\theta$ remains inside that interval, and therefore we are forced to assume this. In terms of the variables $\theta$ and $\tau$ this functional then takes the form

$$
L(\theta, \tau)=\int_{-\ell}^{\ell}\left(\tau+\frac{1}{r} \sin \theta(1-\cos \theta)\right) .
$$

Here we assume rigid loading in shortening and dead loading in twist, i.e. we prescribe the shortening $S$ and the moment $M$, which implies that the tension $T$ and the end rotation $L$ are unknown and to be determined as part of the solution. This loading condition leads to the minimization problem

$$
\min \{E(\theta, \tau)-M L(\theta, \tau): S(\theta)=\sigma\}
$$

for given $\sigma>0$. The tension $T$ has a natural interpretation as a Lagrange multiplier associated with the constraint of $S$.

We can simplify this minimization problem by first minimizing with respect to $\tau$ for fixed $\theta$, from which we find $\tau \equiv M / C$; re-insertion yields the final minimization problem

$$
\min \{F(\theta): S(\theta)=\sigma\}
$$


with

$$
F(\theta)=\frac{B}{2} \int \dot{\theta}^{2}+\frac{B}{2 r^{2}} \int \sin ^{4} \theta-\frac{M}{r} \int \sin \theta(1-\cos \theta) .
$$

We are interested in localized forms of deformation, in which the deformation is concentrated on a small part of the rod and in which boundary effects are to be avoided, and therefore we take an infinitely long rod and consider $\theta, F$, and $S$ to be defined on the whole real line and assume $\theta \rightarrow 0$ as $|s| \rightarrow \infty$.

3.3. Behaviour of minimizers. The Euler-Lagrange equations associated with the minimization problem (7) can be written as a Hamiltonian system with one degree of freedom,

$$
\frac{1}{2} \dot{\theta}^{2}+V(\theta)=H
$$

for a particular $V$. In this system two independent parameters remain, which may be interpreted as a scaled cylinder radius $\tilde{r}=r M / B$ and a combined loading parameter $m=M / \sqrt{B T}$.

Solutions of the original minimization problem are orbits of this Hamiltonian system that are homoclinic to zero, and such orbits have been studied in detail in [11]. Among the findings are

(1) For all values of $\tilde{r}$ ranges of $m$ exist with orbits that are homoclinic to the origin;

(2) At some parameter points these homoclinic orbits 'collide' with saddle points. The saddle points correspond to helical solutions (constant angle $\theta$ ) and close to these collisions the homoclinic orbit has a large region of near-constant angle $\theta$.

In Figure 4 a bifurcation diagram is shown with two such collisions, one at a forward helix $\left(0<\theta<\pi / 2\right.$, at $\left.m=m_{c_{2}}\right)$ and one at a backward helix $(\pi / 2<\theta<\pi$, at $\left.m=m_{c_{1}}\right)$.

In [11] the question of stability of these solutions, both local and global, was left untouched. If we interpret the combined load parameter $m$ as a (reciprocal) tension $T$ (with the moment $M$ fixed) then the nature of the bifurcation diagram in Figure 4, involving as it does the mechanically conjugate variables $S$ and $T$, suggests that in each peak the right curve is locally stable [15]. With two peaks occurring however, this does not allow us to predict where the globally stable solution is located.

In this paper we focus on global energy minimization. Corollary 4.3 below states that for sufficiently large shortening, and when contact effects are neglected, global energy minimizers always intersect themselves. It is this result that forms the main motivation of the analysis of this paper: since energy minimization without appropriate penalization leads to self-intersection, the non-self-intersection condition is necessary for physically acceptable solutions.

3.4. Translation to $(u, \psi)$-coordinates. To study the case in which self-contact is taken into account, it is necessary to properly restrict the class of admissible functions in the minimisation problem (7). In three dimensions a variety of different descriptions of self-contact exists for rods of finite thickness, each with subtle advantages and disadvantages (see e.g. the introduction of [10]). For a rod on a cylinder the situation is simpler, since the freedom of movement is essentially two-dimensional - similar to that of a curve in a plane. We focus on rods of zero 
(a)

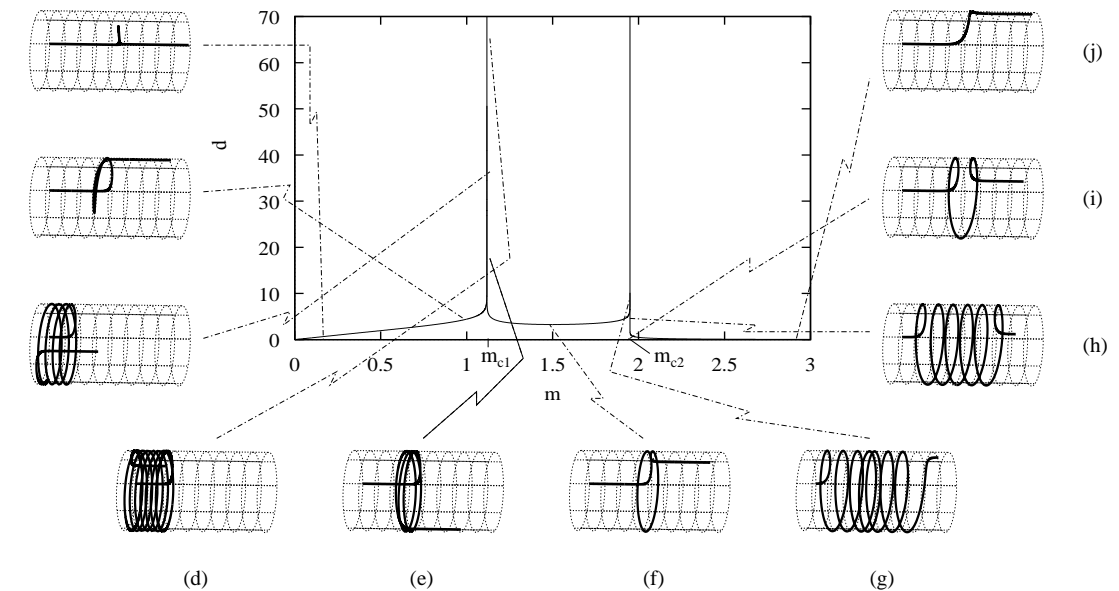

Figure 4. A load-displacement diagram showing shortening $d=$ $S M / B$ of stationary points as a function of the (combined) load $m$ (from [11]). Contact effects are not taken into account. The peaks divide this diagram into three sections. The solutions in the middle section intersect themselves, whilst the solutions on the right do not. The section on the left consists of heteroclinic connections between $\theta=0$ and $\theta=2 \pi$ which are not considered here. For sufficiently large shortening, the rod configuration that has lowest energy is on the self-penetrating branch, as shown by Corollary 4.3 .

thickness, and implement non-self-penetration as non-self-intersection of the centerline. In terms of the unknown $\theta(\cdot)$ as introduced above, this condition can be written as

$$
z\left(s_{1}\right)-z\left(s_{2}\right) \neq 0 \quad \text { for all } \quad s_{1} \neq s_{2} \text { with } \quad \psi\left(s_{1}\right)-\psi\left(s_{2}\right)=0 \quad \bmod 2 \pi,
$$

where we have used the previous equation (6) for $\psi$ and the axial coordinate $z$ :

$$
\dot{\psi}=\frac{1}{r} \sin \theta, \quad \dot{z}=\cos \theta .
$$

We now make the assumption that $z$ can be written as a function of $\psi$, or, equivalently, that $\psi$ is monotonic along the rod. This assumption is satisfied for solutions of the problem without contact having $\theta<\pi$, as given by equation (9). If we include a contact condition of the form (10), then we are unable to prove that $\psi$ is monotonic, and in fact it is conceivable that this monotonicity is only valid for global energy minimizers.

Under the assumption that $z$ can be written as a function of $\psi$, we introduce a dimensionless axial coordinate $\zeta=z / r$, and write ' for differentiation with respect to $\psi$. The functional $F$ in (8) then transforms to

$$
F(\zeta)=\frac{B r}{2} \int_{0}^{T} \frac{\zeta^{\prime \prime 2}}{\left(1+\zeta^{\prime 2}\right)^{\frac{5}{2}}}+\frac{B}{2 r} \int_{0}^{T} \frac{1}{\left(1+\zeta^{\prime 2}\right)^{\frac{3}{2}}}-M \int_{0}^{T} \frac{\sqrt{1+\zeta^{\prime 2}}-\zeta^{\prime}}{\sqrt{1+\zeta^{\prime 2}}},
$$


with shortening

$$
S(\zeta)=r \int_{0}^{T}\left[\sqrt{1+\zeta^{\prime 2}}-\zeta^{\prime}\right] .
$$

Here $[0, T]$, the domain of definition of $\psi$, is a priori unknown, since the ends of the rod are free to move around the cylinder.

In these variables non-self-intersection is easily characterized. Since $\psi$ is monotonic, let us assume it to be increasing (this amounts to an assumption on the sign of the applied moment $M$ ). Admissible functions are defined by the following condition:

$$
\forall \psi \in[0, T-2 \pi]: \zeta(\psi+2 \pi)-\zeta(\psi) \geq 0
$$

Note that it is only necessary to rule out self-intersection after a single turn; if contact exists after multiple turns, contact also exists (potentially elsewhere) after a single turn.

The contact condition (11) is the novel part in this variational problem. In this paper we focus on the effect that this condition has on the minimization problem, and therefore simplify by

- fixing the domain size $T$, and accordingly removing the shortening constraint;

- replacing the mechanically correct boundary conditons $\zeta^{\prime}=\infty$ by a more convenient condition $\zeta^{\prime}=1$.

In terms of the new variables $x=\psi / 2 \pi$ and $u(x)=\zeta^{\prime}(\psi)$ we recover the problem of the introduction.

These boundary conditions can be described as follows. By prescribing $\zeta^{\prime}=u=$ 1 at the ends of the rod we fix the angle between the rod and the centerline to $\pi / 4$. By removing the shortening constraint we allow the ends of the rod to move freely in the axial direction; in contrast, the fixing of $T$ prevents the rod ends from moving tangentially. We believe that these changes have little effect on that part of the rod that is implicated in the contact problem; but this is a topic of current research.

3.5. Zero thickness. The assumption of zero rod thickness can not be relaxed without introducing important changes in the formulation (see Figure 5). At thickness $\epsilon$, the distance in the $\zeta$-direction between two parallel consecutive centerlines in contact is $\epsilon / \sin \theta$, where $\theta$ is the angle between the centerlines and the cylinder axis. Therefore non-zero thickness can not be introduced by simply replacing the right-hand side in (11) by $\epsilon$; the angle of the centerlines is to be taken into account, implying that the right-hand side of (11) will depend on $\zeta^{\prime}$.

To make matters worse, when the centerlines are not parallel, i.e. when $u=\zeta^{\prime}$ is not constant, the minimal-distance connection between two consecutive turns depends on values of $\zeta^{\prime}$ nearby (see [16] for a thorough treatment of the geometry of this issue); it is not clear whether for the present case of a rod on a cylinder any simpler impenetrability condition can be found than the well-known global curvature condition [10].

\section{EXISTEnCE AND THE CONTACT CONDITION}

In this section we state precisely the problem under discussion and show that minimizers exist. We also study the minimization problem without the contact constraint, and show that minimizers will intersect themselves. 


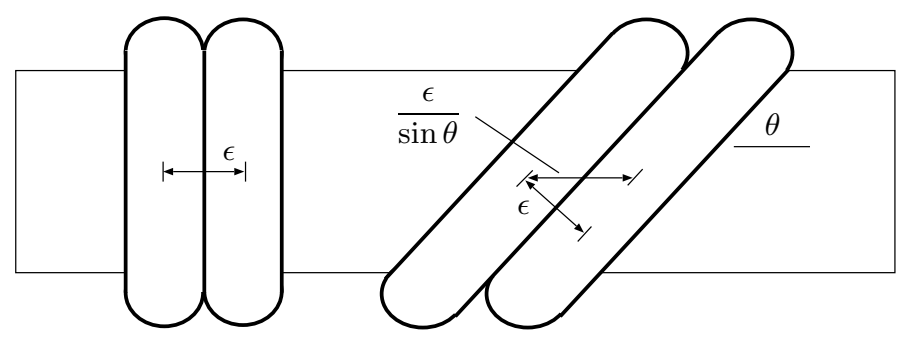

Figure 5. Two configurations of a rod of thickeness $\epsilon$. This illustrates that for rods with positive thickness one cannot simply replace the contact condition $B u \geq 0$ by $B u \geq \epsilon$; a more involved condition is necessary.

Let $U=1+X$, where $X=H_{0}^{1}(0, T)$, and $Y=C([0, T-1])$. Let the functional $F: U \rightarrow \mathbb{R}$ be defined as in the Introduction,

$$
F(u)=\int_{0}^{T}\left[a(u) u^{\prime 2}+b(u)\right],
$$

and introduce the constraint operator $B: U \rightarrow Y$ given by

$$
B(u)(x)=\int_{x}^{x+1} u .
$$

With the set of admissible functions given as

$$
K:=\{u \in U: B(u)(x) \geq 0 \forall x \in[0, T-1]\}
$$

the central problem is

Problem (A): Find a function $u^{*} \in U$ such that

$$
F\left(u^{*}\right)=\min \{F(u): u \in K\} .
$$

We first prove existence of minimizers for Problem (A).

Lemma 4.1. Let $T>0$. Assume that $a(u) \geq a_{0}>0$, and that $b(u)$ is Lipschitz continuous. Then there exists $u^{*} \in K$ such that

$$
F\left(u^{*}\right)=\min \{F(u): u \in K\} .
$$

Proof. Let $\left\{u_{n}\right\} \subset K \subset U$ be a minimizing sequence. We first prove that $\int b\left(u_{n}\right)$ is bounded from below.

Since minimization of $F$ is equivalent to minimization of $F-T b(0)$, we can assume without loss of generality that $b(0)=0$. Using the Lipschitz continuity of $b$ and the Poincaré inequality we have

$$
\left\|b\left(u_{n}\right)\right\|_{L^{1}} \leq c\left\|u_{n}\right\|_{L^{1}} \leq c_{1}\left(T+\left\|u_{n}-1\right\|_{L^{1}}\right) \leq c\left(1+\left\|u_{n}^{\prime}\right\|_{L^{2}}\right) .
$$


Here and below $c$ is a possibly changing constant that does not depend on $n$. Then

$$
\begin{aligned}
\int b\left(u_{n}\right) & \geq-c\left(1+\left\|u_{n}^{\prime}\right\|_{L^{2}}\right) \\
& \geq-c\left(1+\frac{1}{a_{0}}\left(\int a\left(u_{n}\right) u_{n}^{\prime 2}\right)^{\frac{1}{2}}\right) \\
& \geq-c\left(1+\frac{1}{a_{0}}\left(F\left(u_{n}\right)-\int b\left(u_{n}\right)\right)^{\frac{1}{2}}\right) \\
& \geq-c\left(1+\frac{1}{a_{0}}\left(c-\int b\left(u_{n}\right)\right)^{\frac{1}{2}}\right) .
\end{aligned}
$$

Hence

$$
\int b\left(u_{n}\right) \geq-D
$$

for a suitable constant $D$.

Together with the boundedness of $F\left(u_{n}\right),(12)$ implies that $u_{n}$ is bounded in $X$. Hence $\left\{u_{n}\right\}$ contains a subsequence $\left\{u_{n_{m}}\right\}$ that converges weakly in $X$ to a limit $u^{*}$. Since $F$ is lower semicontinuous with respect to weak convergence,

$$
F\left(u^{*}\right) \leq \liminf _{m \rightarrow \infty} F\left(u_{n_{m}}\right)
$$

implying that $u^{*}$ is a minimizer.

As we mentioned in Section 3, if contact is not taken into account-if $F$ is minimized in $U$ rather than in the smaller set $K$-condition. In the theorem below we actually prove a stronger statement. We write $F_{T}$ instead of $F$ to indicate explicitly the dependence on the interval $[0, T]$.

Theorem 4.2 (Minimization without contact condition). Assume that a and $b$ are of class $C^{1}$, and that a is strictly positive. Assume that some $\bar{u}<1$ exists such that

$$
-\infty<\inf _{\mathbb{R}} b<\inf _{u \geq \bar{u}} b .
$$

For each $T>0$, let $u_{T}$ be a minimizer corresponding to the minimization problem on domain $[0, T]$,

$$
\min \left\{F_{T}(u): u \in U\right\} .
$$

Then there exists $c>0$ independent of $T$ such that

$$
\left|\left\{x \in[0, T]: u_{T}(x) \geq \bar{u}\right\}\right| \leq c(1+\sqrt{T}) .
$$

The function $b$ given in (1) achieves its minimum at $u=-\infty$, regardless of the value of $M r / B$; therefore it satisfies the condition (13) for every $\bar{u}<1$.

Corollary 4.3 (Minimizers violate the contact condition). In addition to the conditions of Theorem 4.2, assume that $\bar{u}<0$. If $T$ is sufficiently large, then $B\left(u_{T}\right)(x)<0$ for some $x \in[0, T-1]$.

Proof of Theorem 4.2. We first use a standard argument to give an upper bound on the energy $F_{T}\left(u_{T}\right)$. Choose a $T$-dependent constant $\underline{u}_{T}<\bar{u}$ such that

$$
b\left(\underline{u}_{T}\right)<\inf _{u \geq \bar{u}} b(u) \quad \text { and } \quad 0<b\left(\underline{u}_{T}\right)-\inf _{\mathbb{R}} b \leq T^{-1 / 2} .
$$


For any $T \in \mathbb{R}^{+}$we construct a new continuous symmetric function $\tilde{u}_{T} \in U$ such that $\tilde{u}_{T}=\underline{u}_{T}$ on $[1, T-1]$, and $F_{T}\left(\tilde{u}_{T}\right) \leq C+T b(\underline{u})$, where $C$ does not depend on $T$. Since $u_{T}$ minimizes $F_{T}$, it also follows that

$$
F_{T}\left(u_{T}\right) \leq F_{T}\left(\tilde{u}_{T}\right) \leq C+T b\left(\underline{u}_{T}\right) .
$$

Among other things this inequality implies that for large $T$ a minimizer $u_{T}$ can not be the constant function 1 .

The Euler-Lagrange equation associated with this minimization problem is

$$
-2 a(u) u^{\prime \prime}-a^{\prime}(u) u^{\prime 2}+b^{\prime}(u)=0,
$$

which can also be written als a one-degree-of-freedom Hamiltonian system

$$
-a(u) u^{\prime 2}+b(u)=H .
$$

It follows that for any minimizer $u$,

(1) $b(u(x))=H$ at any stationary point $x$ of $u$;

(2) $b(u(x)) \geq H$ for all $x \in[0, T]$;

(3) $b(1)>H$.

The third statement follows from noting that if $b(1)=H$ then $u \equiv 1$ would be the unique solution of (17).

We now show that any minimizer $u$ is bi-monotonic, i.e. increasing or decreasing away from a minimum or maximum. Suppose instead that $u$ has two internal stationary points, a minimum at $x_{1}$ and a maximum at $x_{2}$; assume for definiteness that $0<x_{1}<x_{2}<T$. Note that $u\left(x_{1}\right)<1<u\left(x_{2}\right)$, since the solution of the Hamiltonian system is a periodic orbit oscillating between the values $u\left(x_{1}\right)$ and $u\left(x_{2}\right)$; the inequality $u\left(x_{1}\right)<1<u\left(x_{2}\right)$ follows from the boundary condition. Now pick a point $x_{12} \in\left(x_{1}, x_{2}\right)$ such that $u\left(x_{12}\right)=1$.

Construct a new function

$$
\tilde{u}(x)= \begin{cases}u(x) & 0 \leq x \leq x_{1} \\ u\left(x_{1}\right) & x_{1} \leq x \leq x_{1}+T-x_{12} \\ u\left(x-T+x_{12}\right) & x_{1}+T-x_{12} \leq x \leq T\end{cases}
$$

Then

$$
\begin{aligned}
F_{T}(\tilde{u}) & =\int_{0}^{x_{1}}\left[a(u) u^{\prime 2}+b(u)\right]+\int_{x_{1}}^{x_{1}+T-x_{12}} b\left(u\left(x_{1}\right)\right)+\int_{x_{1}}^{x_{12}}\left[a(u) u^{\prime 2}+b(u)\right] \\
& =\int_{0}^{x_{12}}\left[a(u) u^{\prime 2}+b(u)\right]+\int_{x_{1}}^{x_{1}+T-x_{12}} b\left(u\left(x_{1}\right)\right) \\
& =\int_{0}^{x_{12}}\left[a(u) u^{\prime 2}+b(u)\right]+H\left(T-x_{12}\right) \\
& <F_{T}(u) .
\end{aligned}
$$

Therefore the assumption of two stationary points is contradicted. Note that by (17) the solution also is symmetric in $[0, T]$. 
We now return to the sequence of functions $u_{T}$. Setting $A=\{x \in[0, T]$ : $\left.u_{T}(x) \geq \bar{u}\right\}$ we have

$$
\begin{aligned}
C+T b\left(\underline{u}_{T}\right) & \geq F_{T}\left(u_{T}\right) \\
& \geq|A| \inf _{u \geq \bar{u}} b(u)+(T-|A|) \inf _{\mathbb{R}} b \\
& =|A|\left(\inf _{u \geq \bar{u}} b(u)-\inf _{\mathbb{R}} b\right)+T \inf _{\mathbb{R}} b,
\end{aligned}
$$

so that

$$
|A|\left(\inf _{u \geq \bar{u}} b(u)-\inf _{\mathbb{R}} b\right) \leq C+T\left(b\left(\underline{u}_{T}\right)-\inf _{\mathbb{R}} b\right) \leq c(1+\sqrt{T}) .
$$

This concludes the proof.

Remark 4.4. By a very similar argument one may show the following statement: if $\min _{\mathbb{R}} b$ is uniquely achieved at some $\bar{u} \in \mathbb{R}$, then

$$
\left\|u_{T}-\bar{u}\right\|_{L^{\infty}(\sqrt{T}, T-\sqrt{T})} \longrightarrow 0 \quad \text { as } \quad T \longrightarrow \infty \text {. }
$$

\section{The Euler-Lagrange Equation}

We characterize the duality $\left(X, X^{\prime}\right)$ by identifying the smooth functions on $[0, T]$ with a dense subset of $X^{\prime}$ via the duality pairing

$$
X^{\prime}\langle\xi, x\rangle_{X}=\int_{0}^{T} \xi x .
$$

Similarly we identify $Y^{\prime}$ with the space of Radon measures $R M([0, T-1])$ via the same duality pairing, defined for smooth functions,

$$
Y^{\prime}\langle\eta, y\rangle_{Y}=\int_{0}^{T} \eta y
$$

Where necessary, we extend Radon measures in $Y^{\prime}$ by zero outside of their domain $[0, T-1]$.

Theorem 5.1. Assume that $a$ and $b$ are globally Lipschitz continuous, and that $a \geq a_{0}>0$. Let $u \in U$ be a solution of Problem $(A)$. Then $u \in W^{2, \infty}(0, T)$ and there exists a Radon measure $f \in Y^{\prime}$ such that

$$
-2 a(u(x)) u^{\prime \prime}(x)-a^{\prime}(u(x)) u^{\prime 2}(x)+b^{\prime}(u(x))=\int_{x-1}^{x} f(s) d s
$$

for almost every $x \in(0, T)$. Moreover $f \geq 0$ and supp $f \subset \omega_{c}$.

Definition 5.2. A function $u \in U$ is called a stationary point if it there exists a Radon measure $f \in Y^{\prime}$, with $f \geq 0$ and supp $f \subset \omega_{c}$, such that (18) is satisfied.

In the rest of the paper we will often drop the arguments in (18) and write

$$
-2 a(u) u^{\prime \prime}-a^{\prime}(u) u^{\prime 2}+b^{\prime}(u)=\int_{x-1}^{x} f .
$$

The proof of Theorem 5.1 follows along the lines of [2]. We fix the function $u$, with contact set $\omega_{c}$ defined in (3), and introduce the cone of admissible perturbations $V$,

$V:=\left\{v \in X: \exists\left\{\varepsilon_{n}\right\}_{n \in \mathbb{N}} \subset \mathbb{R}^{+}, \varepsilon_{n} \rightarrow 0\right.$ such that $\left.B\left(u+\varepsilon_{n} v\right) \geq 0 \forall n \in \mathbb{N}\right\}$.

Lemma 5.3. Let $u$ be a minimizer. Then $F^{\prime}(u) \cdot v \geq 0$ for all $v \in \bar{V}$. 
Proof. For any $v \in V$, the fact $u$ is a minimizer implies that

$$
F\left(u+\varepsilon_{n} v\right)-F(u) \geq 0 \quad \text { for all } n \in \mathbb{N} .
$$

The conditions on $a$ and $b$ imply that $F$ is Fréchet differentiable in $u$ (this follows from the conditions on $a$ and inspection of (20) below), so that

$$
0 \leq F\left(u+\varepsilon_{n} v\right)-F(u)=\varepsilon_{n} F^{\prime}(u) \cdot v+o\left(\varepsilon_{n}\|v\|_{X}\right),
$$

from which it follows that $F^{\prime}(u) \cdot v \geq 0$. Now, given any $v \in \bar{V}$, take a sequence $v_{m} \subset V$ that converges to $v$ in $X$. Since $F^{\prime}(u): X \rightarrow \mathbb{R}$ is a continuous linear operator, $F^{\prime}(u) \cdot v_{m} \rightarrow F^{\prime}(u) \cdot v$. Hence $F^{\prime}(u) \cdot v \geq 0$ for any $v \in \bar{V}$.

$\bar{V}$ can be characterized in a more convenient way:

Lemma 5.4. For any $u \in K$,

$$
\bar{V}=W:=\left\{v \in X: B v \geq 0 \text { on } \omega_{c}\right\} .
$$

We postpone the proof to the end of this section.

$\bar{V}$ is a closed convex cone, with dual cone

$$
\bar{V}^{\perp}=\left\{\gamma \in X^{\prime}:\langle\gamma, v\rangle \geq 0 \quad \forall v \in \bar{V}\right\} .
$$

Let

$$
P=\left\{y \in Y: y \geq 0 \text { on } \omega_{c}\right\} .
$$

This also is a closed convex cone, with dual cone

$$
P^{\perp}=\left\{f \in Y^{\prime}:\langle f, y\rangle \geq 0 \quad \forall y \in P\right\} .
$$

Lemma 5.5. If $f \in P^{\perp}$, then supp $f \subset \omega_{c}$ and $f \geq 0$.

Proof. Given any $y$ with support in $\omega_{c}^{c}, y \in P$ and $-y \in P$. Hence $\langle f, y\rangle=0$ and therefore supp $f \subset \omega_{c}$. Now take $y \in Y$ positive. Then in particular $y \geq 0$ on $\omega_{c}$, and $y \in P$. By definition of $P^{\perp}$ this implies $f \geq 0$.

We now use the following Lemma to characterize $\bar{V}^{\perp}$ in a different way.

Lemma 5.6. Let $Y$ be a Banach space, and $P \subset Y$ a closed convex cone with dual cone $P^{\perp}$. Let $X$ be a second Banach space, and $A: X \rightarrow Y$ a bounded linear operator. Let $K$ be the following cone in $X$ :

$$
K=\{u \in X: A u \in P\} .
$$

Then the dual cone $K^{\perp}$ can be characterized by

$$
K^{\perp}=\left\{A^{T} g \in X^{\prime}: g \in P^{\perp}\right\} .
$$

The proof of this Lemma can be found in [2]. An immediate consequence of Lemma 5.6 is

\section{Corollary 5.7.}

$$
\bar{V}^{\perp}=\left\{B^{T} f \in X^{\prime}: f \in P^{\perp}\right\} .
$$

We now turn to the proof of the main theorem of this section. 
Proof of Theorem 5.1. We have seen that, since $u$ is a minimizer, $F^{\prime}(u) \in \bar{V}^{\perp}$ and

$$
\bar{V}=\left\{v \in X: B(v) \geq 0 \text { on } \omega_{c}\right\}
$$

by Lemmas 5.3 and 5.4. By Corollary 5.7 there exists an $f \in P^{\perp}$ such that $F^{\prime}(u)=$ $B^{T} f$, and by Lemma 5.5 supp $f \subset \omega_{c}$ and $f \geq 0$. The conjugate operator $B^{T}$ is easily seen to be given by

$$
B^{T} \phi(x)=\int_{x-1}^{x} \phi(s) d s
$$

for a smooth function $\phi \in Y^{\prime}$, where $\phi$ is implicitly extended by zero outside of the interval $[0, T-1]$. We use the same notation for a general Radon measure $f \in Y^{\prime}$.

Lastly, direct computation gives

$$
F^{\prime}(u) \cdot v=\int_{0}^{T}\left[2 a(u) u^{\prime} v^{\prime}+a^{\prime}(u) u^{\prime 2} v+b^{\prime}(u) v\right],
$$

and hence we obtain the equation

$$
-2\left[a(u(x)) u^{\prime}(x)\right]^{\prime}+a^{\prime}(u(x)) u^{\prime}(x)^{2}+b^{\prime}(u(x))=\int_{x-1}^{x} f
$$

in the sense of distributions.

We now turn to the statement of regularity. Since $f \in R M([0, T-1])$, the function $g$ (defined in (5)) is uniformly bounded. Since all terms in (21) except the first are in $L^{1}(0, T)$, we have $a(u) u^{\prime} \in W^{1,1}$, and the lower bound on $a$ implies that $u \in W^{2,1}(0, T)$. Since $W^{2,1} \subset W^{1, \infty}$, the second term is now known to be in $L^{\infty}$, and again the lower bound on $a$ is used to obtain $u \in W^{2, \infty}(0, T)$. This regularity of $u$ implies that the distributional equation (21) is also satisfied almost everywhere.

We still owe the reader the proof of Lemma 5.4.

Proof of Lemma 5.4. $\bar{V} \subset W$ : Since $B: X \rightarrow Y$ is continuous, $W$ is closed, and therefore it suffices to show that $V \subset W$. Take any $v \in V$ and $x \in \omega_{c}$. Then $B\left(u+\varepsilon_{n} v\right)(x) \geq 0$, and by definition of $\omega_{c}, B u(x)=0$, implying that $B v(x) \geq 0$. It follows that $v \in W$.

$W \subset \bar{V}:$ First consider $w \in W$ such that $\operatorname{supp}(B w)_{-}$(the support of the negative part of $B w)$ is contained in $\omega_{c}^{c}$. We claim that $w \in V$, for which we have to show that there exists

$$
\left\{\varepsilon_{n}\right\} \subset \mathbb{R}^{+}, \varepsilon_{n} \rightarrow 0 \text {, such that } B\left(u+\varepsilon_{n} w\right) \geq 0 \forall n \in \mathbb{N} .
$$

For $x \in \omega_{c}, B u(x)=0$, and since $B w(x) \geq 0$ we have $B\left(u+\varepsilon_{n} w\right)(x) \geq 0$ for any sequence $\left\{\varepsilon_{n}\right\} \subset \mathbb{R}^{+}$. For the complement $\omega_{c}^{c}$, note that since supp $(B w)_{-}$is compact and contained in the open set $\omega_{c}^{c}$, there exists $\delta>0$ such that $B u \geq \delta>0$ on $\operatorname{supp}(B w)_{-}$. Hence, if $\varepsilon_{n} \leq \delta\|B w\|_{L^{\infty}}^{-1}$, then $B u+\varepsilon_{n} B w \geq 0$ on supp $(B w)_{-}$. Note that $B u \geq 0$ on $[0, T-1]$, and $B w \geq 0$ on $\left(\operatorname{supp}(B w)_{-}\right)^{c}$. This means that $B u+\varepsilon_{n} B w \geq 0$ on $\omega_{c}^{c}$. Together with $B w \geq 0$ on $\omega_{c}$, this implies $w \in V$.

Finally, consider a general $w \in W$. Fix a smooth function $\phi \in X$ with $\phi>0$ on $(0, T)$; note that $B \phi \geq c>0$. We approximate $w$ by the function $w_{\varepsilon}:=w+\varepsilon \phi$. We claim that $\operatorname{supp}\left(B w_{\varepsilon}\right)_{-} \subset \omega_{c}^{c}$ for sufficiently small $\varepsilon>0$. It then follows that $w_{\varepsilon} \in V$ and $w_{\varepsilon} \rightarrow w$, implying that $w \in \bar{V}$. 
To prove the claim, note that $w \in X \subset L^{\infty}$. Hence $B w$ is Lipschitz continuous, with Lipschitz constant $2\|w\|_{L^{\infty}}$. Hence, for small enough $\varepsilon$,

$$
\begin{aligned}
B w_{\varepsilon}(x) & =B w(x)+\varepsilon B \phi(x) \\
& \geq B w(y)+\varepsilon B \phi(y)-3|| w \|_{L^{\infty}}|x-y| .
\end{aligned}
$$

Suppose $B w_{\varepsilon}(x)<0$ and $y \in \omega_{c}$. Then $B w(y) \geq 0$, and by (22),

$$
-\varepsilon B \phi(y)>-\left.3|| w\right|_{L^{\infty}}|x-y|,
$$

or

$$
|x-y|>\frac{\varepsilon B \phi(y)}{3\|w\|_{L^{\infty}}} .
$$

Therefore $d\left(\operatorname{supp}(B w)_{-}, \omega_{c}\right)>C \varepsilon$ for a suitable $C>0$. Hence supp $\left(B w_{\varepsilon}\right)_{-} \subset \omega_{c}^{c}$ for small enough $\varepsilon$, which proves the claim.

\section{Characterization of Stationary points}

For this section we assume that the conditions of Theorem 5.1 are met.

Lemma 6.1. Let $u$ be a stationary point, and let $g$ be defined as in (5).

(1) For all $x \in \omega_{c}, u(x)=u(x+1)$ and $u^{\prime}(x) \leq u^{\prime}(x+1)$.

(2) If $\omega_{c}$ contains an interval $\left[x_{0}, x_{1}\right]$, then

- $u^{\prime}(x)=u^{\prime}(x+1)$ for all $x \in\left(x_{0}, x_{1}\right)$;

- $u^{\prime \prime}(x)=u^{\prime \prime}(x+1)$ and $g(x)=g(x+1)$ for almost all $x \in\left(x_{0}, x_{1}\right)$.

This lemma imposes an interesting form of periodicity on the solution and the right-hand side $g$. Although the constraint is a non-local one, on an interval of contact of length $L$ the solution actually only has the degrees of freedom of an interval of length one; the other values follow from this assertion.

Proof. Since $x \in \omega_{c}$,

$$
\int_{x}^{x+1} u=0
$$

Hence, since $B u(x)=\int_{x}^{x+1} u \in W^{3, \infty}$, and $B u \geq 0$,

$$
0=\frac{d}{d x} \int_{x}^{x+1} u=u(x+1)-u(x),
$$

and

$$
0 \leq \frac{d^{2}}{d x^{2}} \int_{x}^{x+1} u=u^{\prime}(x+1)-u^{\prime}(x) .
$$

If $B u=0$ on $\left[x_{0}, x_{1}\right]$, then the inequality above becomes an equality a.e. on the interior $\left(x_{0}, x_{1}\right)$, implying that

$$
u^{\prime}(x)=u^{\prime}(x+1) \text { on }\left(x_{0}, x_{1}\right) .
$$

The periodicity of $u^{\prime \prime}$ and $g$ now follow from (18).

Lemma 6.2. Let $u$ be a stationary point, and assume that $\omega_{c}$ contains an interval I. Then

$$
\int_{x}^{x+1} g
$$

is constant on Int $I$. 
Proof. By Lemma $6.1 u(x)=u(x+1)$ for all $x \in I$, and $u^{\prime \prime}(x)=u^{\prime \prime}(x+1)$ a.e. on $I$. In addition, $u^{\prime}(x)=u^{\prime}(x+1)$ for all $x \in \operatorname{Int} I$. Hence

$$
x \mapsto \int_{x}^{x+1}\left[-2 a(u) u^{\prime \prime}-a^{\prime}(u) u^{\prime 2}+b^{\prime}(u)\right]
$$

is constant on $I$. But by (18), (23) is equal to

$$
\int_{x}^{x+1} \int_{s-1}^{s} f=\int_{x}^{x+1} g .
$$

The following two lemmas and the theorem that follows are essential in determining the structure of the right-hand side $g$ and therefore of the measure $f$. The main argument is the following. The function $g$ has no reason to be monotonic; its derivative in $x$ equals $f(x)-f(x-1)$, and although $f$ is a positive measure this difference may be of either sign. However, if for instance a left end point $x_{0}$ of $\omega_{c}$ is flanked by a non-contact interval $\left(x_{0}-1, x_{0}\right)$, then the measure $f$ is zero on that interval, and the function $g$ is non-decreasing on $\left(x_{0}, x_{0}+1\right)$. It is this argument, repeated from both sides, that allows us to determine completely the structure of the function $g$ and the underlying measure $f$.

Notation Let $\left[x_{0}, x_{1}\right] \subset \omega_{c}$. Define

$$
p \equiv x_{1}-x_{0}(\bmod 1)
$$

and

$$
P=\min \left\{n \in \mathbb{N}: n \geq x_{1}-x_{0}\right\} .
$$

Throughout the rest of this paper $\tau$ is the translation operator defined by

$$
(\tau u)(x)=u(x+1) .
$$

Lemma 6.3. Let $u$ be a stationary point, such that $\omega_{c}$ contains an interval $\left[x_{0}, x_{1}\right]$. Assume furthermore that

$$
\operatorname{supp} f \cap\left(x_{0}-1, x_{0}\right)=\emptyset .
$$

Then

(1) if $x_{1}-x_{0} \in \mathbb{N}$, then $g$ does not decrease on each of the subintervals

$$
\left(x_{0}+i, x_{0}+i+1\right), i=0,1, \ldots, P ;
$$

(2) if $x_{1}-x_{0} \notin \mathbb{N}$, then $g$ does not decrease on each of the subintervals

$$
\left(x_{0}+i, x_{0}+i+1\right), i=0,1, \ldots, P-1,
$$

nor does it on

$$
\left(x_{0}+P, x_{1}+1\right) \text {. }
$$

Proof. On $\left(x_{0}, x_{0}+1\right)$,

$$
g^{\prime}=f-\tau^{-1} f \stackrel{(27)}{=} f \geq 0,
$$

and therefore $g$ is non-decreasing on $\left(x_{0}, x_{0}+1\right)$. By Lemma 6.1, $g(x)=g(x+1)$ for almost all $x \in\left(x_{0}, x_{1}\right)$. This implies that on each consecutive interval $\left(x_{0}+i, x_{0}+\right.$ $i+1), i=1, \ldots, P-1, g$ does not decrease. By the same reasoning, if $x_{1}-x_{0} \in \mathbb{N}$, then this also holds for $\left(x_{0}+P, x_{0}+P+1\right)=\left(x_{1}, x_{1}+1\right)$. If not, then it holds for $\left(x_{0}+P, x_{1}+1\right)$. 
Remark 6.4. Let $u$ be a stationary point. Define the mirror image $v(x)=u(T-x)$, and $h(x)=f(T-x-1)$. Then $(v, h)$ solves

$$
\left\{\begin{array}{l}
-2 a(v) v^{\prime \prime}-a^{\prime}(v) v^{\prime 2}+b^{\prime}(v)=\int_{x-1}^{x} h \\
v(0)=v(T)=1
\end{array}\right.
$$

and hence is also a stationary point.

Applying Lemma 6.3 to $(v, h)$ yields for $(u, f)$ :

Lemma 6.5. Let $u$ be a stationary point such that $\omega_{c}$ contains an interval $\left[x_{0}, x_{1}\right]$. Assume furthermore that

$$
\text { supp } f \cap\left(x_{1}+1, x_{1}+2\right)=\emptyset .
$$

(1) if $x_{1}-x_{0} \in \mathbb{N}$, then $g$ does not increase on each of the subintervals

$$
\left(x_{0}+i, x_{0}+i+1\right), i=0,1, \ldots, P
$$

(2) if $x_{1}-x_{0} \notin \mathbb{N}$, then $g$ does not increase on each of the subintervals

$$
\left(x_{0}+p+i, x_{0}+p+i+1\right), i=0,1, \ldots, P-1,
$$

nor does it on

$$
\left(x_{0}, x_{0}+p\right) .
$$

To combine the previous two Lemmas, let

$$
\begin{array}{lll}
X_{i}=x_{0}+i, & i=0, \ldots, P, \\
Y_{i}=x_{0}+p+i, & i=0, \ldots, P .
\end{array}
$$

Theorem 6.6. Let $u$ be a stationary point such that the contact set $\omega_{c}$ contains an interval $\left[x_{0}, x_{1}\right]$. Suppose that

$$
\operatorname{supp} f \cap\left\{\left(x_{0}-1, x_{0}\right) \cup\left(x_{1}+1, x_{1}+2\right)\right\}=\emptyset .
$$

Then there exists $G \in \mathbb{R}$ such that

(1) if $x_{1}-x_{0} \in \mathbb{N}$, then $g \equiv G$ on $\left(x_{0}, x_{1}+1\right)$, and

$$
\left.f\right|_{\left(x_{0}-1, x_{1}+2\right)}=G \sum_{i=0}^{P} \delta\left(x-X_{i}\right) .
$$

(2) if $x_{1}-x_{0} \notin \mathbb{N}$, then

$$
g(x)=\left\{\begin{array}{lll}
g_{1} & \text { on }\left[X_{i}, Y_{i}\right], & i=0, \ldots, P \\
g_{2} & \text { on }\left[Y_{i}, X_{i+1}\right], & i=0, \ldots, P-1,
\end{array}\right.
$$

and

$$
\left.f\right|_{\left(x_{0}-1, x_{1}+2\right)}=\sum_{i=0}^{P-1} a_{i} \delta\left(x-X_{i}\right)+b_{i} \delta\left(x-Y_{i}\right),
$$

where $a_{i}=\left(G-\frac{i}{P}\right) g_{1}$ and $b_{i}=\frac{G+i}{P} g_{1}$, and

$$
\begin{gathered}
g_{1}:=\frac{G P}{P+1-p} \in\left(\frac{P G}{P+1}, G\right), \\
g_{2}:=\frac{G(P+1)}{P+1-p}=\frac{P+1}{P} g_{1} \in\left(G, \frac{(P+1) G}{P}\right) .
\end{gathered}
$$


Proof. (1) $x_{1}-x_{0} \in \mathbb{N}$.

By Lemma $6.3, g$ does not decrease on the intervals $\left(X_{i}, X_{i+1}\right), i=0,1, \ldots, P$, and by Lemma $6.5 \mathrm{~g}$ does not increase on these intervals either. Hence $g$ is constant on each interval. By Lemma 6.2 the constant is the same on each interval, i.e. that $g \equiv G$ on $\left(x_{0}, x_{1}+1\right)$. This also implies that within the interval $\left(x_{0}-1, x_{1}+2\right)$, $f$ can only have support in the points $x_{0}=X_{0}, X_{1}, \ldots, X_{P}=x_{1}$, yielding the formula for $f$ in the statement of the theorem.

(2) $x_{1}-x_{0} \notin \mathbb{N}$.

Combining Lemma 6.3 and 6.5 , we find that $g$ is constant on each interval $\left(X_{i}, Y_{i}\right)$ and $\left(Y_{i}, X_{i+1}\right), i=0,1, \ldots, P-1$, and on $\left(X_{P}, Y_{P}\right)$. By Lemma $6.1, g(x)=g(x+1)$ for almost all $x \in\left(x_{0}, x_{1}\right)$, and hence $g$ takes three values, 0 , and $g_{1}$ and $g_{2}$ (say) on $\left(x_{0}, x_{1}+1\right)$. We choose $g=g_{1}$ on $\left(X_{i}, Y_{i}\right), i=0,1, \ldots, P$, and $g=g_{2}$ on the intervals inbetween, $\left(Y_{i}, X_{i+1}\right), i=0,1, \ldots, P-1$; outside of the interval $\left(x_{0}, x_{1}\right)$, $g$ vanishes. By Lemma 6.2,

$$
G=\int_{x}^{x+1} g=\int_{x}^{x+p} g_{1}+\int_{x+p}^{x+1} g_{2}=p g_{1}+(1-p) g_{2} \text {. }
$$

Either $g_{1}=g_{2}=G$ or $g_{1}<G<g_{2}$. The first case implies that $g \equiv G$ on $\left[x_{0}, x_{1}+1\right]$. This implies that $f$ does not only have support in $X_{0}, X_{1}, \ldots, X_{P-1}$, but by reasoning for the mirror image $(v, h)$ it also implies that $f$ has support in $Y_{0}, Y_{1}, \ldots, Y_{P-1}=x_{1}$. This is impossible. Hence $g_{1}<G<g_{2}$. The support of $f$ on $\left(x_{0}-1, x_{1}+2\right)$ is now seen be to limited to the set given in the statement of the Theorem.

Thus we conclude that $f$ is a sum of delta functions, but we still have to determine the weights $a_{i}$ and $b_{i}$. Since $f=0$ on $\left(x_{0}-1, x_{0}\right)$, we have $g_{1}=g\left(x_{0}+\right)=f\left(x_{0}\right)$. Here we abuse notation, and write $f(x)$ for the weight of the Dirac delta function at $x$. Now we have the following recurrence relations:

$$
\begin{aligned}
& f\left(X_{i}\right)+f\left(Y_{i}\right)=g_{2}, \\
& f\left(Y_{i}\right)+f\left(X_{i+1}\right)=g_{1},
\end{aligned}
$$

for $i=0,1, \ldots, P-1$. Solving this system we obtain

$$
\begin{aligned}
& f\left(X_{i}\right)=f\left(X_{0}\right)-i\left(g_{2}-g_{1}\right)=g_{1}-i\left(g_{2}-g_{1}\right), \\
& f\left(Y_{i}\right)=(i+1)\left(g_{2}-g_{1}\right) .
\end{aligned}
$$

In addition, since $x_{1}=Y_{P-1}, f\left(x_{1}\right)=P\left(g_{2}-g_{1}\right)$. On the other hand, $g_{1}=$ $h\left(T-x_{1}-1\right)=f\left(x_{1}\right)$. This implies

$$
g_{2}=\frac{P+1}{P} g_{1}
$$

To conclude,

and

$$
a_{i}=f\left(X_{i}\right)=\left(G-\frac{i}{P}\right) g_{1},
$$

By Lemma 6.2,

$$
b_{i}=f\left(Y_{i}\right)=\frac{i+1}{P} g_{1} .
$$

which yields

$$
p g_{1}+(1-p) \frac{P+1}{P} g_{1}=G
$$

$$
p=P\left(1-\frac{G}{g_{1}}\right)+1 .
$$


Solving for $g_{1}$ now yields all required results.

As we will see in the next Section, the contact set of $u$ is connected in many important cases. Hence Theorem 6.6 allows us to give concise expressions for $g$ in cases that $\omega_{c}$ is an interval of positive length (using the Heaviside function $H$ ):

Corollary 6.7. If the contact set is an interval of positive length, then $g$ equals the explicit function

$$
\begin{aligned}
& g\left(x ; x_{0}, x_{1}, G\right)= \\
& \quad \begin{cases}H\left(x-x_{0}\right)-H\left(x-x_{1}-1\right) & \text { if } x_{1}-x_{0} \in \mathbb{N}, \\
g_{1}\left(H\left(x-x_{0}\right)-H\left(x-x_{1}-1\right)\right)+ & \text { if } x_{1}-x_{0} \notin \mathbb{N} .\end{cases}
\end{aligned}
$$

Here the coefficients $g_{1,2}$ are computed from $x_{0}, x_{1}$, and $G$ by (24), (25), (30). and (31).

Figure 6 shows examples of both cases. For the remaining two cases of a stationary point that has a single or no contact point, $g$ is immediately clear: with a single contact point,

$$
g(x)= \begin{cases}m & \text { on }\left[x_{0}, x_{0}+1\right] \\ 0 & \text { otherwise }\end{cases}
$$

for a suitable constant $m \geq 0$, while when there is no contact then obviously $g \equiv 0$.

\section{The CONTACT SET IS AN INTERVAL}

In order to extract more information on the right-hand side $g$ and the measure $f$ than that given by Theorem 5.1 we study two cases. In the first case we assume that the operator given by the left-hand side in (18) satisfies a version of the classical comparison principle. In the second case we restrict ourselves to global minimizers.

Definition 7.1. Let $N$ be a (non)linear operator on $U . N$ is said to satisfy the comparison principle if for any $\left[x_{0}, x_{1}\right] \subset[0, T]$,

$$
\left.\begin{array}{l}
N u_{1} \leq N u_{2}, \\
u_{1}\left(x_{0}\right) \leq u_{2}\left(x_{0}\right), \\
u_{1}\left(x_{1}\right) \leq u_{2}\left(x_{1}\right),
\end{array}\right\} \Longrightarrow u_{1} \leq u_{2} \text { on }\left[x_{0}, x_{1}\right] .
$$

See e.g. [9] or [17] for a general exposition. Operators of the type considered here, i.e.

$$
N u:=-2 a(u) u^{\prime \prime}-a^{\prime}(u) u^{\prime 2}+b^{\prime}(u),
$$

may fail to satisfy the comparison principle for two reasons. First, the zero-order term $b^{\prime}(u)$ need not be increasing in $u$; for instance, the operator $u \mapsto-u^{\prime \prime}-u$ does not satisfy the comparison principle on any interval of length $2 \pi$ or more. In a slightly more subtle manner, the prefactor $a(u)$ of the second-order derivative may also invalidate the comparison principle; see e.g. [9, Section 10.3] for an example.

We conjecture that the 'true' rod functions $a$ and $b$ given in (1) do not give rise to a comparison principle: $b^{\prime}$ is not monotonic, suggesting that on sufficiently large intervals the principle will fail.

We first prove a lemma that will be used in both cases. 

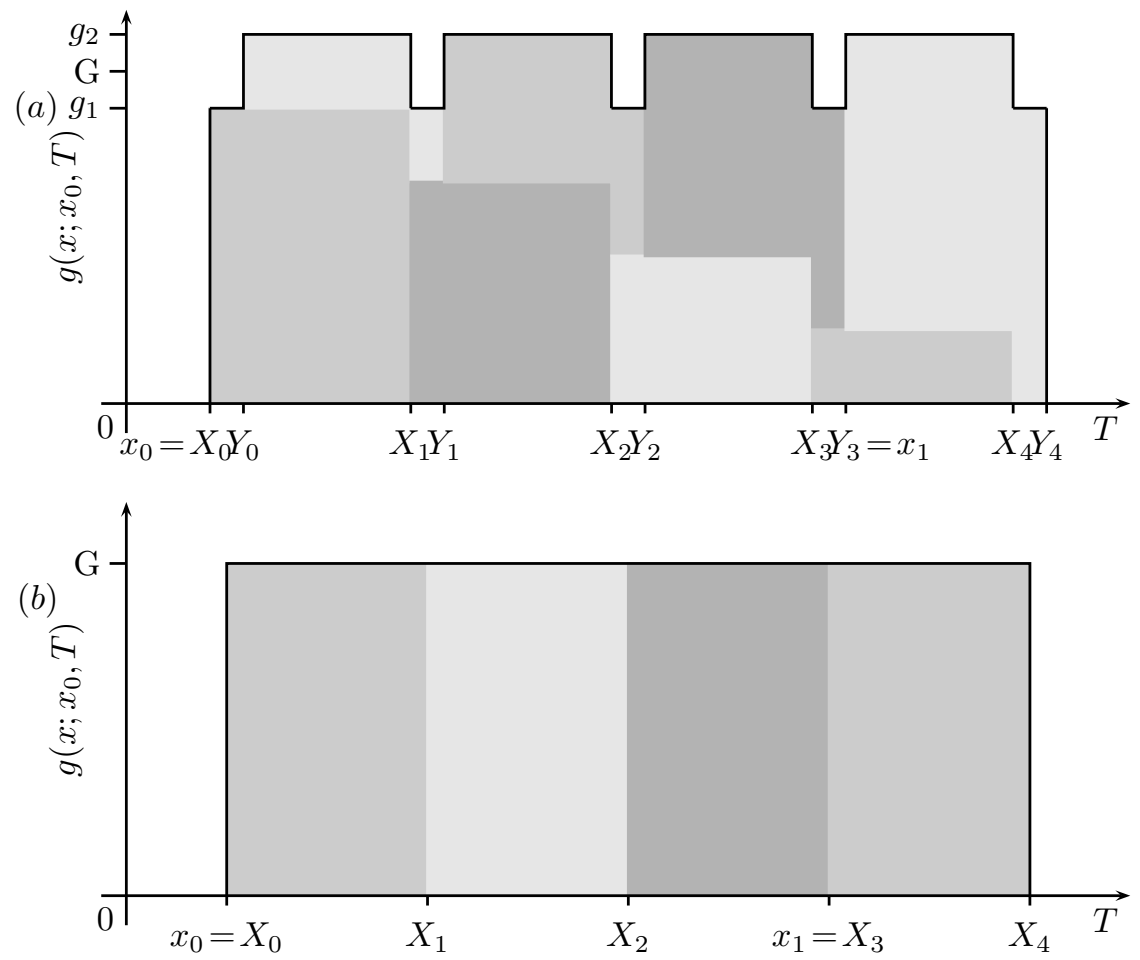

FiguRE 6. A generic picture of $g\left(x ; x_{0}, T\right)$ for $T-x_{0} \notin \mathbb{N}(a)$ and for $T-x_{0} \in \mathbb{N}(b)$. The light gray shaded areas represent the contributions of the individual delta functions of the corresponding Radon measures $f$. As an example, in $(b) f$ consists of four Dirac deltas, all with mass $G$, at $x_{0}=X_{0}, x_{0}+1=X_{1}, \ldots, x_{0}+3=X_{3}$.

Lemma 7.2. Let $u$ be a stationary point such that $x_{1}, x_{2} \in \omega_{c}$. Assume that

$$
\left(x_{1}, x_{2}\right) \cap \omega_{c}=\emptyset .
$$

Then

$$
\int_{x_{1}}^{x_{2}} u=\int_{x_{1}+1}^{x_{2}+1} u
$$

and for any $m \in\left(0, x_{2}-x_{1}\right)$,

$$
\int_{x_{1}}^{x_{1}+m} u<\int_{x_{1}+1}^{x_{1}+1+m} u \text { and } \quad \int_{x_{2}-m}^{x_{2}} u>\int_{x_{2}-m+1}^{x_{2}+1} u .
$$

Proof. Since $\left(x_{1}, x_{2}\right) \cap \omega_{c}=\emptyset$,

$$
\int_{x_{1}+m}^{x_{1}+m+1} u>0
$$


for all $m \in\left(0, x_{2}-x_{1}\right)$, which implies

$$
\begin{aligned}
\int_{x_{1}+1}^{x_{1}+m+1} u-\int_{x_{1}}^{x_{1}+m} u & =\int_{x_{1}}^{x_{1}+1} u+\int_{x_{1}+1}^{x_{1}+m+1} u-\int_{x_{1}}^{x_{1}+m} u \\
& =\int_{x_{1}+m}^{x_{1}+m+1} u>0 .
\end{aligned}
$$

The other two assertions are handled similarly.

Theorem 7.3. Let $u$ be a stationary point, and assume that

$$
N u:=-2 a(u) u^{\prime \prime}-a^{\prime}(u) u^{\prime 2}+b^{\prime}(u)
$$

satisfies the comparison principle. Then $\omega_{c}$ is connected.

Proof. We proceed by contradiction. Since $\omega_{c}$ is closed, non-connectedness implies the existence of $x_{1}, x_{2} \in \omega_{c}$ such that $\left(x_{1}, x_{2}\right) \cap \omega_{c}=\emptyset$.

Set $v=u-\tau u$. Then $v\left(x_{1}\right)=v\left(x_{2}\right)=0$ by Lemma $6.1, \int_{x_{1}}^{x_{2}} v=0$ by (34), and

$$
\int_{x_{1}}^{x_{1}+m} v<0 \text { for all } 0<m<x_{2}-x_{1}
$$

by (35). Hence there exists an $\bar{x} \in\left(x_{1}, x_{2}\right)$ such that $v(\bar{x})=0$.

From $\left(x_{1}, x_{2}\right) \cap \omega_{c}=\emptyset$ it follows that supp $f \cap\left(x_{1}, x_{2}\right)=\emptyset$. Hence $g=\int_{x-1}^{x} f$ is a decreasing function on $\left(x_{1}, x_{2}\right)$ and $\tau g$ is an increasing function on this interval by previous arguments. Hence $g-\tau g$ is a decreasing function on $\left(x_{1}, x_{2}\right)$. There are three possibilities, each leading to a contradiction with the comparison principle.

Case 1: $g \geq \tau g$ on $\left(x_{1}, x_{2}\right)$. On $\left(x_{1}, \bar{x}\right)$,

$$
\left\{\begin{array}{l}
N u=g \geq \tau g=N \tau u, \\
u\left(x_{1}\right)=\tau u\left(x_{1}\right), \\
u(\bar{x})=\tau u(\bar{x}) .
\end{array}\right.
$$

By the comparison principle, $u \geq \tau u$ on $\left(x_{1}, \bar{x}\right)$, i.e. $v \geq 0$. But this contradicts (37).

Case 2: there exists an $\tilde{x}$ such that $g \geq \tau g$ on $\left(x_{1}, \tilde{x}\right)$ and $g \leq \tau g$ on $\left(\tilde{x}, x_{2}\right)$. If $\tilde{x} \geq x_{1}$, the same argument applies. If $\tilde{x}<\bar{x}$, we consider $\left(\bar{x}, x_{2}\right)$ instead, and apply the same argument. Now we conclude $v \leq 0$ on $\left(\bar{x}, x_{2}\right)$. But observe that from $\int_{x_{1}}^{\bar{x}} v<0$ by $(37)$ and $\int_{x_{1}}^{x_{2}} v=0$ we have $\int_{\bar{x}}^{x_{2}} v>0$, which again implies a contradiction.

Case 3: $g \leq \tau g$ on $\left(x_{1}, x_{2}\right)$. Again we obtain a contradiction from considering the interval $\left(\bar{x}, x_{2}\right)$.

For the second case we limit ourselves to global minimizers. The results of this theorem do apply to the functions $a$ and $b$ given in (1).

Theorem 7.4. Let $u$ be a minimizer. Assume that $a$ and $b$ are of class $C^{1}$ and that $a$ is strictly positive. Then $\omega_{c}$ is connected.

Proof. As in the proof of Theorem 7.3 we assume that there exist $x_{1}, x_{2} \in \omega_{c}$ with $\left(x_{1}, x_{2}\right) \cap \omega_{c}=\emptyset$ to force a contradiction. Then

$$
\text { supp } f \cap\left(x_{1}, x_{2}\right)=\emptyset \text {, }
$$


and hence $g$ is a decreasing function on $\left(x_{1}, x_{2}\right)$, and an increasing function on $\left(x_{1}+1, x_{2}+1\right)$. Now consider the following two new functions

$$
v(x)= \begin{cases}u(x) & \text { on }\left[0, x_{1}\right] \\ u(x+1) & \text { on }\left[x_{1}, x_{2}\right] \\ u(x) & \text { on }\left[x_{2}, T\right]\end{cases}
$$

and

$$
w(x)= \begin{cases}u(x) & \text { on }\left[0, x_{1}+1\right] \\ u(x-1) & \text { on }\left[x_{1}+1, x_{2}+1\right] \\ u(x) & \text { on }\left[x_{2}+1, T\right]\end{cases}
$$

Both are admissible, i.e. $v, w \in K$ : they are continuous by Lemma 6.1 , implying that $v, w \in X$, and the fact that $B v, B w \geq 0$ follows from Lemma 7.2. In fact we need certain strict inequalities, which we derive after introducing some notation.

The functions $v$ and $w$ are minimizers. To show this, write

$$
F\left(\left.u\right|_{\left[x_{1}, x_{2}\right]}\right)=\int_{x_{1}}^{x_{2}}\left[a(u) u^{\prime 2}+b(u)\right] .
$$

Then since $u$ is a minimizer, and since $u$ and $v$ only differ on $\left[x_{1}, x_{2}\right]$,

$$
F\left(\left.u\right|_{\left[x_{1}, x_{2}\right]}\right) \leq F\left(\left.v\right|_{\left[x_{1}, x_{2}\right]}\right)=F\left(\left.u\right|_{\left[x_{1}+1, x_{2}+1\right]}\right)
$$

and similarly

$$
F\left(\left.u\right|_{\left[x_{1}+1, x_{2}+1\right]}\right) \leq F\left(\left.w\right|_{\left[x_{1}+1, x_{2}+1\right]}\right)=F\left(\left.u\right|_{\left[x_{1}, x_{2}\right]}\right) .
$$

This implies that

$$
F\left(\left.u\right|_{\left[x_{1}, x_{2}\right]}\right)=F\left(\left.u\right|_{\left[x_{1}+1, x_{2}+1\right]}\right),
$$

and that $F(u)=F(v)=F(w)$. Every minimizer is also a stationary point, and hence for $v$ and $w$ there exist positive Radon measures $f_{v}$ and $f_{w}$ such that $\operatorname{supp} f_{v} \subset \omega_{c}(v)$ and $\operatorname{supp} f_{w} \subset \omega_{c}(w)$. We also denote $g_{v}(x)=\int_{x-1}^{x} f_{v}$ and $g_{w}(x)=\int_{x-1}^{x} f_{w}$.

For any $x \in\left(x_{1}, x_{2}\right)$,

$$
\int_{x}^{x+1} u>0
$$

Let first $x_{2} \geq x_{1}+1$. Then for any $x \in\left(x_{1}-1, x_{1}\right)$,

$$
\begin{aligned}
\int_{x}^{x+1} v & =\int_{x}^{x_{1}} v+\int_{x_{1}}^{x+1} v \\
& =\int_{x}^{x_{1}} u+\int_{x_{1}+1}^{x+2} u \\
& >\int_{x}^{x_{1}} u+\int_{x_{1}}^{x+1} u \quad \text { by Lemma } 7.2 \\
& =\int_{x}^{x+1} u \geq 0 .
\end{aligned}
$$

For any $x \in\left(x_{1}, x_{2}-1\right)$ the same is true:

$$
\int_{x}^{x+1} v=\int_{x+1}^{x+2} u>0
$$


since $x+1<x_{2}$, which allows us to use (39). Now let $x_{2}<x_{1}+1$. Then for any $x \in\left(x_{1}-1, x_{2}-1\right)$, we can repeat the first argument above to conclude

$$
\int_{x}^{x+1} v>0
$$

Combining these statements we find

$$
\int_{x}^{x+1} v>0 \text { for all } x \in\left(x_{1}-1, x_{2}-1\right)
$$

which implies $\omega_{c}(v) \cap\left(x_{1}-1, x_{2}-1\right)=\emptyset$. Hence supp $f_{v} \cap\left(x_{1}-1, x_{2}-1\right)=$ $\emptyset$. But since $u$ and $v$ conincide on $\left[0, x_{1}\right]$, we have $\left.g_{v}\right|_{\left[0, x_{1}\right]}=\left.g_{u}\right|_{\left[0, x_{1}\right]}$, so that supp $f_{u} \cap\left(x_{1}-1, x_{2}-1\right)=\emptyset$. Combined with (38), this implies that $\left.g_{u}\right|_{\left[x_{1}, x_{2}\right]}$ is constant. By symmetry the same is true for $\left.g_{u}\right|_{\left[x_{1}+1, x_{2}+1\right]}$. Note that if $x_{2}>x_{1}+1$, then the overlap implies that the two constants are the same; for the other case we now prove this.

Define $z=u-\tau u$; the function $z$ solves the equation

$$
\begin{aligned}
-2 a(u) z^{\prime \prime}=g_{u}-\tau g_{u}+\left\{a^{\prime}(u) u^{2}-a^{\prime}(\tau u)(\tau u)^{\prime 2}\right\} & \\
- & \left\{b^{\prime}(u)-b^{\prime}(\tau u)\right\}+\{2 a(u)-a(\tau u)\}(\tau u)^{\prime \prime}
\end{aligned}
$$

on the interval $\left(x_{1}, x_{2}\right)$. Of the right-hand side, we have seen above that the term $g_{u}-\tau g_{u}$ is constant on $\left(x_{1}, x_{2}\right)$; let us suppose it non-zero for the purpose of contradiction. The function $z$ is of class $C^{1}$, and both $z$ and $z^{\prime}$ vanish at $x=x_{1,2}$. Therefore the assumed regularity on $a$ and $b$ implies that the expressions between braces are continuous on $\left[x_{1}, x_{2}\right]$ and zero at $x=x_{1,2}$. The sign of the right-hand side of (40) is therefore determined by $g_{u}-\tau g_{u}$, and most importantly, is the same at both ends $x_{1}$ and $x_{2}$; therefore the sign of $z$, at $x=x_{1}+$ and $x=x_{2}-$, is also the same. This contradicts the following consequence of Lemma 7.2:

$$
\int_{x_{1}}^{x_{1}+m} z<0 \quad \text { and } \quad \int_{x_{2}-m}^{x_{2}} z>0 \quad \text { for all } 0<m<x_{2}-x_{1} .
$$

This leaves $g_{u}=\tau g_{u}$ on $\left(x_{1}, x_{2}\right)$. But then, by uniqueness of the initial-value problem, $u(x)=u(x+1)$ for all $x \in\left[x_{1}, x_{2}\right]$, and $\left[x_{1}, x_{2}\right] \subset \omega_{c}$, contrary to our assumption that $\left(x_{1}, x_{2}\right) \cap \omega_{c}=\emptyset$.

\section{SyMmetry}

In the introduction we raised the question whether the stationary points or minimizers inherit the symmetry of the formulation, or to put it differently, whether non-symmetric solutions exist.

For the discussion of this question it is useful to introduce an equivalent formulation of the Euler-Lagrange equation (18) similar to the Hamiltonian-systems formulation used in the proof of Theorem 4.2. For the length of this section we assume that Theorem 6.6 applies and therefore that there is a single contact interval $\left[x_{0}, x_{1}\right]$. by

By multiplying (18) with $u^{\prime}$ and integrating one finds that the function $H$, defined

$$
H:=-a(u) u^{\prime 2}+b(u)-g u,
$$

is piecewise constant, and that $H$ and $g$ jump at the same values of $x$. The function $g$ takes three values on $[0, T]$, these being the values $g_{1}$ and $g_{2}$ introduced 
in Theorem 6.6, and the value $g_{0}=0$ outside of the extended contact interval $\left[x_{0}, x_{1}+1\right]$. (Note that $g_{1}$ and $g_{2}$ may be equal). We claim that $H$ also takes three values, $H_{0}, H_{1}$, and $H_{2}$, and that these values correspond to those of $g$, i.e. that the pair $(g, H)$ takes three values $\left(0, H_{0}\right),\left(g_{1}, H_{1}\right)$, and $\left(g_{2}, H_{2}\right)$ (although it may happen that $\left.\left(g_{1}, H_{1}\right)=\left(g_{2}, H_{2}\right)\right)$. Then

To prove this claim, first consider the case of $p>0$, where $p$ is defined as in (24).

$$
\left.\left.u\right|_{\left(x_{0}, x_{0}+p\right)} \equiv u\right|_{\left(x_{0}+1, x_{0}+1+p\right)} \quad \text { and }\left.\left.\quad g\right|_{\left(x_{0}, x_{0}+p\right)} \equiv g\right|_{\left(x_{0}+1, x_{0}+1+p\right)}
$$

by Lemma 6.1 and (29). Therefore $H$ is the same on these two intervals. Repeating this argument for all subintervals of $\left[x_{0}, x_{1}+1\right]$ of the form $\left(x_{0}+k, x_{0}+k+p\right)$ and $\left(x_{0}+k+p, x_{0}+k+1\right)$ we find that $H$ takes two values on the interval $\left[x_{0}, x_{1}+1\right]$, $H_{1}$ and $H_{2}$, and that these coincide with the values $g_{1}$ and $g_{2}$ of $g$.

When $p=0$, a similar argument yields that $H$ takes only one value on $\left[x_{0}, x_{1}+1\right]$ (as does $g$ ).

A consequence of this characterization of $H$ is the following lemma:

Lemma 8.1. Under the conditions and notation of Theorem 6.6,

$$
u\left(x_{0}\right)=u\left(x_{0}+p\right)=u\left(x_{0}+1\right)=u\left(x_{0}+1+p\right)=\cdots=u\left(x_{1}+1\right) .
$$

Proof. When $p=0$ the statement follows from Lemma 6.1. For $p>0$, note that at any of the interior jump points, i.e. at all jump points except $x_{0}$ and $x_{1}+1$, we have $[H]=-[g] u$ where $[H]= \pm\left(H_{2}-H_{1}\right)$ and $[g]= \pm\left(g_{2}-g_{1}\right)$. Regardless of the sign this equation has only one solution $u$. For the remaining two points $x_{0}$ and $x_{1}+1$ the result follows from Lemma 6.1.

We still need to show that the value of $H$ is the same on both sides of the extended contact interval $\left[x_{0}, x_{1}+1\right]$, so that we can define the value $H_{0}$ unambiguously. If one of the ends of this interval equals 0 or $T$ there is nothing to prove; we therefore assume that $\min \left\{x_{0}, T-x_{1}-1\right\} \geq d>0$. Now multiply (18) with the function

$$
v(x)= \begin{cases}\frac{x}{d} u^{\prime}(x) & 0<x<d \\ u^{\prime}(x) & d \leq x \leq T-d \\ \frac{T-x}{d} u^{\prime}(x) & T-d<x<T\end{cases}
$$

and integrate to find

$$
-\frac{1}{d} \int_{0}^{d} H+\frac{1}{d} \int_{T-d}^{T} H=0 .
$$

Since $H$ is constant on $(0, d)$ and on $(T-d, T)$ the two constant values are equal; we then define $H_{0}$ to be this value.

We now turn to the implications of this characterization of solutions $(u, g)$ and the associated pseudo-Hamiltonian function $H$.

Theorem 8.2. Let $u$ be a stationary point with a single contact interval $\left[x_{0}, x_{1}\right]$. Let $p$ be given as in (24), and define the set of jump points $J=\left\{x_{0}, x_{0}+p, x_{0}+\right.$ $\left.1, x_{0}+1+p, \ldots, x_{1}+1\right\}$.

(1) There exists $\alpha \in \mathbb{R}$ such that at any $x \in J, u^{\prime}(x)= \pm \alpha$.

Now assume that $b$ is non-decreasing on $[1, \infty)$.

(2) If the operator $N$ given in (36) satisfies the comparison principle, then $u$ is symmetric on $[0, T]$. 
(3) If $u$ is a minimizer with $u^{\prime}\left(x_{0}\right)=-u^{\prime}\left(x_{1}+1\right)$, then $u$ is symmetric on $[0, T]$.

Proof. For the first part write

$$
u^{\prime 2}=\frac{b(u)-g u-H}{a(u)},
$$

and note that by the proof of Lemma 8.1 the sum $g u+H$ is continuous.

For the second part, note that by Lemma $8.1 u$ has the same value on each end of the interval $\left[x_{0}, x_{0}+p\right]$ (if $p>0$ ) or $\left[x_{0}, x_{0}+1\right]$ (if $p=0$ ). By the uniqueness that follows from the comparison principle the function $u$ is symmetric on this interval. By repeating this argument over all subintervals of $\left[x_{0}, x_{1}+1\right]$ we find that $u$ is symmetric on $\left[x_{0}, x_{1}+1\right]$.

The functions $u_{1}(t):=u\left(x_{0}-t\right)$ and $u_{2}(t):=u\left(x_{1}+1+t\right)$, therefore, have the same zeroth and first derivatives at $t=0$; they satisfy the same equation (41) (note that $H$ is symmetric on $\left[x_{0}, x_{1}+1\right]$ ); therefore the two functions are equal as long as they both exist. This implies that lack of symmetry must stem from a difference in domain of definition of $u_{1}$ and $u_{2}$ for $t>0$.

We claim that neither $u_{1}$ nor $u_{2}$ has an interior maximum. Assuming this claim, the assertion of the theorem follows since the monotonicity of $u_{1,2}$ then implies that the boundary condition $u_{1,2}(t)=1$ has at most one solution $t$.

Now assume that $u_{1}$ has a maximum at $t_{1}>0$. The function $u_{1}$ is solution of the Hamiltonian system (41), where $H$ and $g$ are constant for $t>0$. As in the proof of Theorem 4.2, therefore $u_{1}\left(t_{1}\right)>1$. Choose a bounded interval $I \subset[0, \infty)$ such that $u>1$ on Int $I$ and $u(\partial I)=1$.

The reduced functional $\tilde{F}(v)=\int_{I}\left[a(v) v^{\prime 2}+b(v)\right]$ has a global minimizer $\tilde{v}$ in the class of functions $v$ satisfying $v(\partial I)=1$. From studying the perturbation $v \mapsto \min \{v, 1\}$ and using the monotonicity of $b$ it follows that $\tilde{v} \leq 1$ on $I$. By the comparison principle this is the only stationary point of $\tilde{F}$, a conclusion that contradicts the fact that $u_{1}$ is a different stationary point.

For the third part, first note that the support of the continuous function $x \mapsto$ $\int_{x}^{x+1} u$ is $\left[x_{0}, x_{1}\right]$; therefore

for every $\epsilon>0$ there exists $\delta>0$ such that any perturbation $v$ with $d\left(\operatorname{supp} v,\left[x_{0}, x_{1}+1\right]\right)>\epsilon$ is admissible provided $\|v\|_{L^{\infty}}<\delta$.

We will use this below.

The assumption on the derivatives places us in the same position as above: the functions $u_{1}(t):=u\left(x_{0}-t\right)$ and $u_{2}(t):=u\left(x_{1}+1+t\right)$ are equal as long as they both exist. Again we will show that neither may have an interior maximum, but by a different argument.

Assume that $u_{1}$ has a maximum. By defining $t_{1}=x_{0}$ the boundary condition on $u$ takes the form $u_{1}\left(t_{1}\right)=1$. Pick

$$
\max \left\{1, u_{1}(0)\right\}<\beta<\max \left\{u_{1}(t): 0 \leq t \leq t_{1}\right\}
$$

and define the set $S=\left\{t \in\left[0, t_{1}\right]: u_{1}(t) \geq \beta\right\}$; we can assume that for $\epsilon=\inf S>0$ we have $\max \left\{u_{1}(t): 0 \leq t \leq t_{1}\right\}-\beta<\delta$ for the associated $\delta$ given above.

Now define $v(t)=\min \left\{\beta, u_{1}(t)\right\}$. The function $v$ is admissible by construction; it differs from $u_{1}$ only on the set $S$, and therefore the difference in energy is given 
by (with a slight abuse of notation)

$$
F(v)-F\left(u_{1}\right)=\int_{S}\left[-a\left(u_{1}\right) u_{1}^{2}+b(\beta)-b\left(u_{1}\right)\right]<0 .
$$

This contradicts the assumption of minimality.

The conditions of Theorem 8.2 are quite sharp. We demonstrate this with two examples.

Example 1: $b$ is decreasing on $[1, \infty)$. It is relatively straightforward to construct a non-symmetric stationary point by choosing an appropriate function $b$ that is decreasing on $[1, \infty)$, thus showing that part 2 of Theorem 8.2 is sharp.

Take a symmetric stationary point $u$ for which $u \leq 1$ on $[0, T], u^{\prime}(T)>0$, and for which the contact set is bounded away from $x=T$ (see the next section for examples). Close to $x=T$, the function $u$ satisfies

$$
u^{\prime 2}=\frac{b(u)-H}{a(u)}
$$

for some $H \in \mathbb{R}$, and since $u^{\prime}(T)>0, b(1)>H$. Now change $b(u)$ for $u>1$ such as to have (for instance) $b(2)=H$, and continue the solution $u$ past $x=T$. By construction $u(T+\tilde{T})=2$, for some $\tilde{T}>0$, and $u^{\prime}(T+\tilde{T})=0$; by symmetry then $u(T+2 \tilde{T})=1$. The new function $u$ defined on the domain $[0, t+2 \tilde{T}]$ is a non-symmetric stationary point (Figure 7).

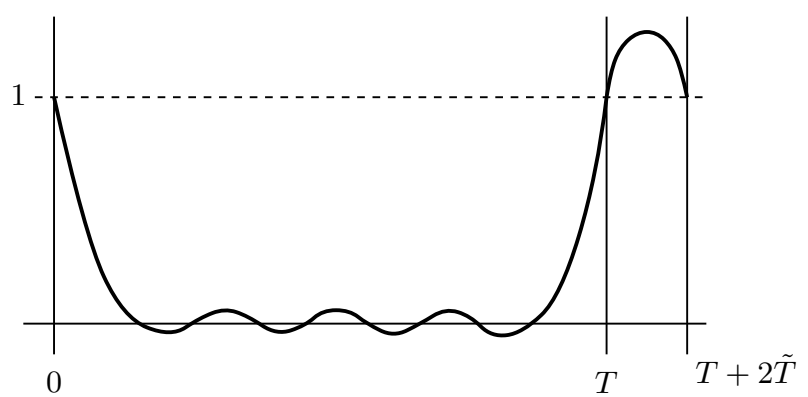

FiguRE 7. A non-symmetric stationary point can be constructed by defining $b(u)$ appropriately for $u>1$.

Example 2: equal (non-opposite) derivatives on $\partial \omega_{c}$. For certain functions $b$ and domains $[0, T]$ global minimization favours breaking of symmetry. We demonstrate this for the functional

$$
F(u)=\int\left[u^{\prime 2}+\alpha\left(1-u^{2}\right)^{2}\right]
$$

where $\alpha$ will be chosen appropriately. We consider the functional $F$ on functions $u:[0,1] \rightarrow \mathbb{R}$ with boundary conditions $u(0)=u(1)=0$; although this is slightly different from the setup in the rest of the paper, it simplifies the argument, and the extension to a more general situation is intuitively clear.

We will show that

$$
\inf \left\{F(u): \int u \geq 0\right\}<\inf \left\{F(u): \int u \geq 0 \text { and } u \text { is symmetric }\right\} .
$$


The infimum on the right-hand side is bounded from below,

$$
F(u) \geq(1-\alpha c / 2) \int u^{\prime 2}+\alpha
$$

by the Poincaré inequality

$$
\int_{0}^{d} u^{2} \leq c d^{2} \int_{0}^{d} u^{\prime 2} \quad \text { for all } u \text { with } u(0)=0 \text { and } \int u=0 .
$$

The function $v(x)=a+\cos (b(1-x / d))$ is optimal in this inequality, where $a \simeq$ 0.22 and $b \simeq 4.49$ are determined by the boundary condition $v(0)=0$ and the integral condition $\int v=0$. The Poincaré constant equals $c \simeq 0.0495$. Note that for symmetric functions $u$ we may take $d=1 / 2$.

At the function $w(x)=\sin 2 \pi x$ the functional $F$ has the value $F(w)=2 \pi^{2}+$ $3 \alpha / 8$. For all $\alpha \in\left(16 \pi^{2} / 5,2 / c\right] \simeq(31.6,40.3]$ therefore

$$
F(w)=2 \pi^{2}+3 \alpha / 8<\alpha \leq \inf \left\{F(u): \int u \geq 0 \text { and } u \text { is symmetric }\right\},
$$

which demonstrates (42).

The reason for this preference for asymmetry can be recognized in the constant in the Poincaré inequality (43) (see Figure 8). For symmetric functions the relevant class is $\left\{u:[0,1 / 2] \rightarrow \mathbb{R}: u(0)=\int u=0\right\}$, and for more general functions $\left\{u:[0,1] \rightarrow \mathbb{R}: u(0)=u(1)=\int u=0\right\}$. For this latter class the Poincaré coefficient is achieved by the function $w$ above with the value $\bar{c}=1 / 4 \pi^{2} \simeq 0.0253$, which is larger than $c(1 / 2)^{2}=0.0124$.
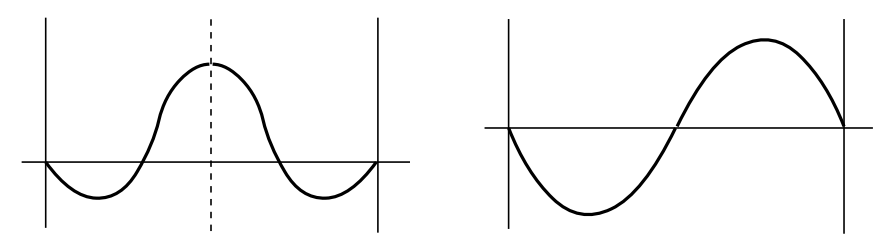

FIGURE 8. Under symmetry conditions the effective domain, the domain on which $\int u=0$, is half the actual domain size. Equivalently, more (costly) oscillations are necessary.

\section{NumericAl Simulations}

In this section we describe in detail our numerical simulations of stationary points of $F$ under constraint, i.e. of solutions of

$$
\begin{aligned}
& -2 a(u) u^{\prime \prime}-a^{\prime}(u) u^{\prime 2}+b^{\prime}(u)=\int_{x-1}^{x} f, \\
& u(0)=u(T)=1 \\
& \text { supp } f \subset \omega_{c}, \\
& f \text { a positive Radon measure, } \\
& \int_{x}^{x+1} u \geq 0 \quad \forall x \in[0, T-1] .
\end{aligned}
$$


We concentrate on the case in which the solution is symmetric and the contact set is non-empty, and we use the fact that the right-hand side in the differential equation can be characterised explicitly (see (33)). We further simplify by replacing the inequality (48) by the condition that the function $x \mapsto \int_{x}^{x+1} u$ has a second-degree zero at $x=x_{0}$, leading to the new system in the unknowns $\left(u, x_{0}, G\right)$

$$
\begin{aligned}
& -2 a(u) u^{\prime \prime}-a^{\prime}(u){u^{\prime}}^{2}+b^{\prime}(u)=g\left(x ; x_{0}, T-x_{0}-1, G\right), \\
& u(0)=u(T)=1, \\
& u\left(x_{0}\right)=u\left(x_{0}+1\right), \\
& \int_{x_{0}}^{x_{0}+1} u=0 .
\end{aligned}
$$

For brevity we shall write $\bar{g}\left(x ; x_{0}, T, G\right)$ for $g\left(x ; x_{0}, T-x_{0}-1, G\right)$.

Lemma 9.1. Assume that the operator on the left-hand side of (49) satisfies the comparison principle. Then any solution of problem (44-48) with non-empty contact set is also a solution of (49-52); vice versa, any solution of (49-52) is also a solution of $(44-48)$.

Proof. Since the implication (44-48) $\Longrightarrow(49-52)$ follows by construction, it suffices to show the opposite implication; in fact, since an admissible measure $f$ can be constructed from any $\bar{g}\left(x ; x_{0}, T, G\right)$, it is sufficient to show that solutions of (49-52) satisfy

We show slightly more, namely that

$$
\int_{x}^{x+1} u \geq 0 \quad \forall x \in[0, T-1] .
$$

$$
\int_{x}^{x+1} u=0 \quad \forall x \in\left[x_{0}, T-x_{0}-1\right]
$$

and that

$$
\int_{x}^{x+1} u>0 \quad \forall x \notin\left[x_{0}, T-x_{0}-1\right] .
$$

The function $u$ is symmetric by Theorem 8.2. Since $u\left(x_{0}\right)=u\left(x_{0}+1\right)$,

$$
u\left(x_{0}\right)=u\left(x_{0}+1\right)=u\left(T-x_{0}\right)=u\left(T-x_{0}-1\right)=: \bar{u} .
$$

Set $u_{1}(x)=u\left(x_{0}+x\right)$, and $u_{2}(x)=u\left(x_{0}+x+1\right)$ for all $x \in\left[0, T-2 x_{0}-1\right]$. By construction, $\bar{g}\left(x ; x_{0}, T, G\right)=\bar{g}\left(x+1 ; x_{0}, T, G\right)$ for all $x \in\left[x_{0}, T-x_{0}-1\right]$. Hence, if we set $h(x)=\bar{g}\left(x+x_{0} ; x_{0}, T, G\right)$, for all $x \in\left[0, T-2 x_{0}-1\right]$, then $u_{1}$ and $u_{2}$ both satisfy

$$
\begin{aligned}
& -2 a(v) v^{\prime \prime}-a^{\prime}(v) v^{\prime 2}+b^{\prime}(v)=h, \\
& v(0)=v\left(T-2 x_{0}-1\right)=\bar{u},
\end{aligned}
$$

By uniqueness, $u_{1}=u_{2}$ on $\left[0, T-2 x_{0}-1\right]$. In terms of $u$ this means $u(x)=u(x+1)$ for all $x \in\left[x_{0}, T-x_{0}-1\right]$. But that implies that

$$
\int_{x}^{x+1} u=0 \quad \forall x \in\left[x_{0}, T-x_{0}-1\right] .
$$

It remains to be shown that

$$
\int_{x}^{x+1} u>0 \quad \forall x \notin\left[x_{0}, T-x_{0}-1\right] .
$$


By symmetry we only show this for $x<x_{0}$. Let $u_{p}$ and $g_{p}$ be the 1 -periodic extrapolation of $\left.u\right|_{\left[x_{0}, x_{0}+1\right]}$ and $\left.g\right|_{\left[x_{0}, x_{0}+1\right]}$; note that $\int_{x}^{x+1} u_{p}=0$ for every $x$. For $x<x_{0}$,

$$
-2 a(u) u^{\prime \prime}-a^{\prime}(u){u^{\prime}}^{2}+b^{\prime}(u)=0<g_{p}=-2 a\left(u_{p}\right) u_{p}^{\prime \prime}-a^{\prime}\left(u_{p}\right){u_{p}^{\prime}}^{2}+b^{\prime}\left(u_{p}\right),
$$

implying that $u>u_{p}$ for $x=x_{0}-$ and therefore also (53) for $x=x_{0}-$. If $u$ and $u_{p}$ intersect again at some $\tilde{x}<x_{0}$, then the comparison principle and (54) imply that $u \leq u_{p}$ on $\left[\tilde{x}, x_{0}\right]$, in contradiction with the previous statement. This concludes the proof.

We discuss two different ways of calculating solutions of the problem (49-52).

9.1. Continuation. We implemented a strategy of continuation of solutions, using the continuation package AUTO [7], and we chose the simple case

$$
a(u)=\frac{1}{2}, \quad b(u)=\frac{1}{2}(u+1)^{2} .
$$

To implement system (49-52) in AUTO, we divide [0,T] into three subdomains, $\left[0, x_{0}\right]$, $\left[x_{0}, x_{0}+1\right]$ and $\left[x_{0}+1, T\right]$ and specify the equations

$$
\begin{aligned}
& \left.\begin{array}{r}
-u_{1}^{\prime \prime}\left(x_{1}\right)+u_{1}\left(x_{1}\right)+1=\bar{g}\left(x_{1} ; x_{0}, T, G\right) \\
x_{1}^{\prime}=1
\end{array}\right\} \text { on }\left[0, x_{0}\right], \\
& \left.\begin{array}{r}
-u_{2}^{\prime \prime}\left(x_{2}\right)+u_{2}\left(x_{2}\right)+1=\bar{g}\left(x_{2} ; x_{0}, T, G\right) \\
x_{2}^{\prime}=1
\end{array}\right\} \text { on }\left[x_{0}, x_{0}+1\right], \\
& \left.\begin{array}{r}
-u_{3}^{\prime \prime}\left(x_{3}\right)+u_{3}\left(x_{3}\right)+1=\bar{g}\left(x_{3} ; x_{0}, T, G\right), \\
x_{3}^{\prime}=1
\end{array}\right\} \text { on }\left[x_{0}+1, T\right],
\end{aligned}
$$

with boundary conditions

$$
\begin{gathered}
u_{1}(0)=1, \\
u_{1}\left(x_{0}\right)=u_{2}\left(x_{0}\right), u_{1}^{\prime}\left(x_{0}\right)=u_{2}^{\prime}\left(x_{0}\right), \\
u_{2}\left(x_{0}\right)=u_{3}\left(x_{0}\right), u_{2}^{\prime}\left(x_{0}\right)=u_{3}^{\prime}\left(x_{0}\right), \\
u_{3}(T)=1, \\
u_{2}\left(x_{0}\right)=u_{2}\left(x_{0}+1\right), \\
x_{1}(0)=0, x_{2}\left(x_{0}\right)=x_{0}, x_{3}\left(x_{0}+1\right)=x_{0}+1 .
\end{gathered}
$$

and integral condition

$$
\int_{x_{0}}^{x_{0}+1} u_{2}=0 .
$$

Note that in (56)-(58) we have added trivial equations in order to solve for the $x_{i}$ variables, which are required in the evaluation of $\bar{g}\left(x ; x_{0}, G, T\right)$.

There are still some technicalities that have to be overcome: AUTO is not wellequipped to handle systems with a discontinuous right-hand side, such as the function $g\left(x ; x_{0}, G, T\right)$ that is supplied here. We remedy this by using a low-order 
method for all simulations, and we smooth the function $g$ given in (33) by substituting arctans for Heaviside functions:

$$
\begin{aligned}
& \tilde{g}\left(x ; x_{0}, T, G\right)= \\
& \left\{\begin{array}{c}
\frac{g_{1}}{\pi}\left(\arctan \left(A\left(x-x_{0}\right)\right)-\arctan \left(A\left(x-T-x_{0}\right)\right)\right) \quad \text { if } T-2 x_{0} \notin \mathbb{N}, \\
\quad+\frac{\left(g_{2}-g_{1}\right)}{\pi} \sum_{i=1}^{P}\left[\arctan \left(A\left(x-X_{i}\right)\right)-\arctan \left(A\left(x-Y_{i}\right)\right)\right], \\
\frac{1}{\pi}\left(\arctan \left(A\left(x-x_{0}\right)\right)-\arctan \left(A\left(x-T-x_{0}\right)\right)\right) \quad \text { if } T-2 x_{0} \in \mathbb{N},
\end{array}\right.
\end{aligned}
$$

where $X_{i}$ and $Y_{i}$ are as in (28). In the limit $A \rightarrow \infty, \tilde{g}\left(x ; x_{0}, T, G\right)$ converges pointwise to $\bar{g}\left(x ; x_{0}, T, G\right)$.

There are nine differential equations with ten boundary conditions and one integral condition. This means that we expect to specify three free parameters to obtain a one-parameter curve of solutions. These are $T, x_{0}$, and an additional parameter $\beta$. It worked well to choose the freedom in $\beta$ in modulating the values of $g_{1,2}$ :

$$
\tilde{g}_{1}=g_{1}+\beta \quad \text { and } \quad \tilde{g}_{2}=g_{2}+\beta
$$

One may prove a priori that $\beta=0$ by remarking that

$$
\int_{x_{0}}^{x_{0}+1} \tilde{g}=\int_{x_{0}}^{x_{0}+1}\left[-u^{\prime \prime}+u+1\right]=1,
$$

and using (32) to find

$$
1=\int_{x_{0}}^{x_{0}+1} \tilde{g}=p\left(g_{1}+\beta\right)+(1-p)\left(g_{2}+\beta\right)=1+\beta .
$$

We have found no other role for $\beta$ than to accommodate for small numerical inaccuracies due to the discontinuous right-hand side. In all simulations $\beta \simeq 10^{-4}$.

We have validated the code by comparing solutions from AUTO with explicit solutions. An example is given in Figure 9.

As we have seen in the discussion at the beginning of this Section, as $T$ becomes larger the minimizer $u$ has to have a contact point, and for large enough values even a full interval of contact. The point $x_{0}$, the leftmost point of contact, is determined as part of the solution; one may wonder how this point depends on $T$. For operators $N$ that satisfy the comparison principle, it is straightforward to prove that $x_{0}$ remains bounded for all $T$. Moreover, for the operator under consideration here, as $T \rightarrow \infty, x_{0} \rightarrow \log (2+\sqrt{3})$. These two phenomena are illustrated in Figure 10.

Since $\left|g_{1}-g_{2}\right| \rightarrow 0$ as $P$ (and therefore $T$ ) increases, $g$ becomes constant in the limit of large $T$. By the comparison principle, $u$ does the same, implying that $F(u) / T \rightarrow 1$. The start of the convergence to 1 is shown in Figure 11 .

9.2. Directly solving the boundary-value problem. Computing solutions of the rod equations - rather than the simpler problem (55) - using AUTO has proved difficult, for reasons that we do not understand well. Instead, a boundary-value problem solver from Matlab was used to create Figure 3. Set

$$
L u=-\frac{2 u^{\prime \prime}}{4 \pi^{2}\left(1+u^{2}\right)^{\frac{5}{2}}}+\frac{5 u u^{\prime 2}}{4 \pi^{2}\left(1+u^{2}\right)^{\frac{7}{2}}}-\frac{3 u}{r^{2}\left(1+u^{2}\right)^{\frac{5}{2}}}+\frac{\alpha}{\left(1+u^{2}\right)^{\frac{3}{2}}} .
$$




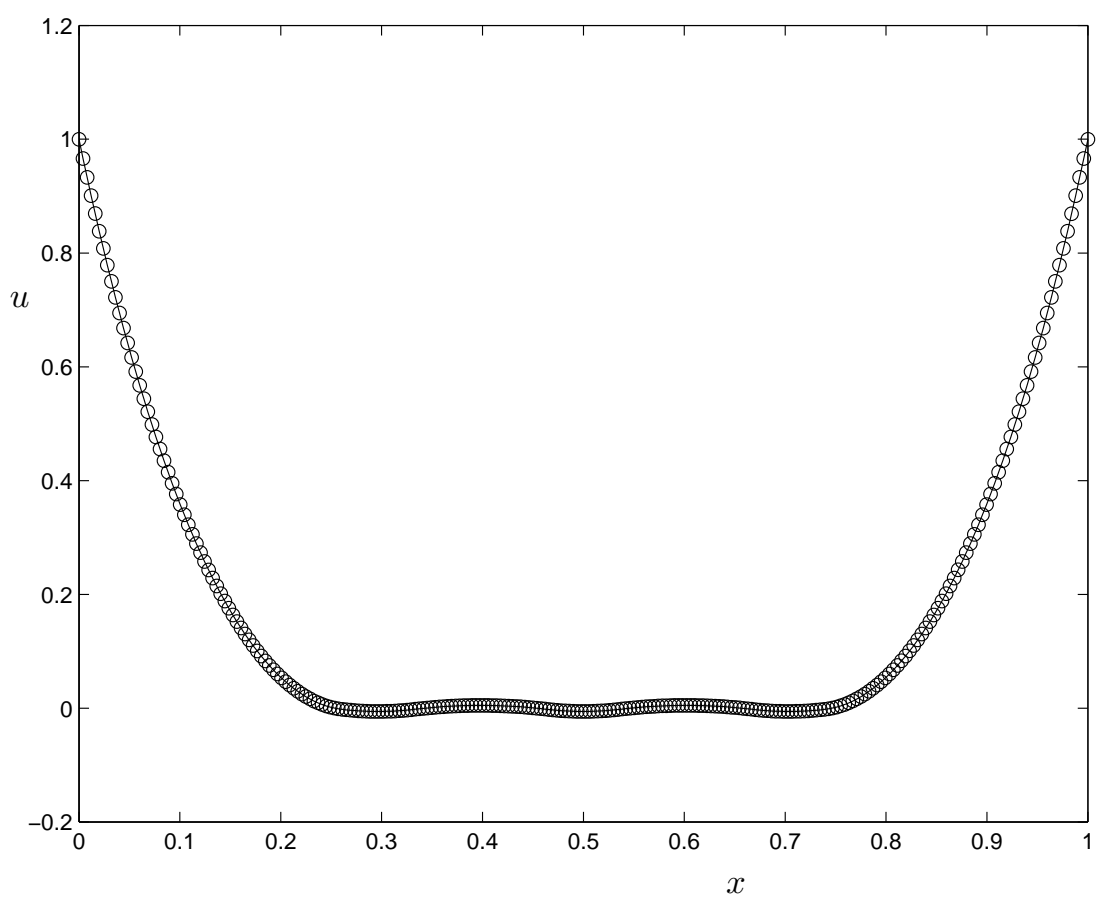

Figure 9. A comparison of a solution of system (56)-(60) produced with AUTO (o symbols) to an explicit solution, for a generic value of $T$ (here scaled to 1 ): $T=4.91635$. In this simulation $A=1000$.

To find a solution of

$$
L u=g\left(x ; x_{0}, T, G\right), \quad u\left(x_{0}\right)=u\left(x_{0}+1\right), \quad \int_{x_{0}}^{x_{0}+1} u=0
$$

for a generic value of $T$ (large enough) we construct a two-parameter shooting problem. Fix $G$ and $x_{0}$ and consider the boundary-value problem

$$
\begin{array}{ll}
L u_{1}=0 & \text { on }\left[0, x_{0}\right] \\
L u_{2}=g_{1} & \text { on }\left[x_{0}, x_{0}+p\right] \\
L u_{3}=g_{2} & \text { on }\left[x_{0}+p, x_{0}+1\right], \\
L u_{u}=g_{1} & \text { on }\left[x_{0}+1, x_{0}+1+p\right] \\
L u_{5}=0 & \text { on }\left[x_{0}+1+p, \tilde{T}\right]
\end{array}
$$




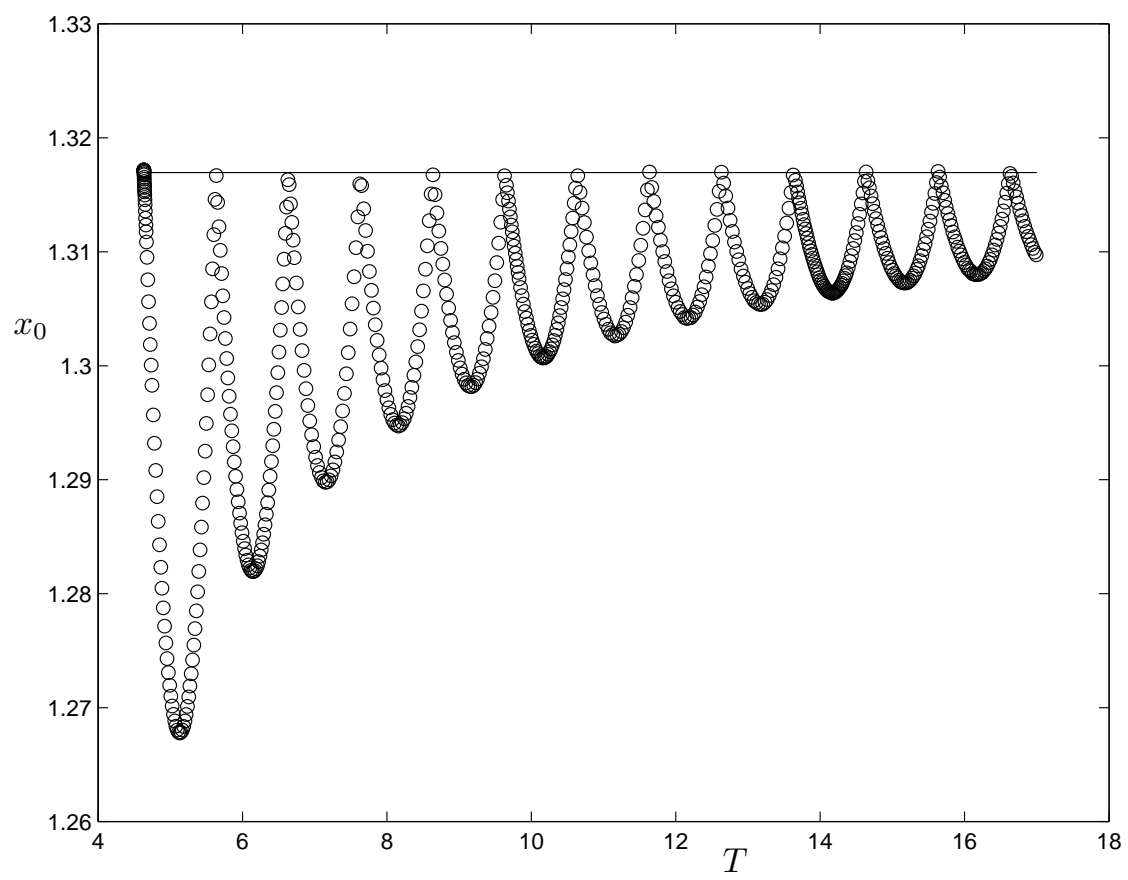

FiguRE 10. Behaviour of $x_{0}$ as a function of domain size $T$ for system (56)-(60) computed with AUTO. As $T$ grows, $x_{0}$ remains bounded and converges to $\log (2+\sqrt{3})$ (horizontal line).

with boundary conditions

$$
\begin{gathered}
u_{1}(0)=1, \\
u_{1}\left(x_{0}\right)=u_{2}\left(x_{0}\right), u_{1}^{\prime}\left(x_{0}\right)=u_{2}^{\prime}\left(x_{0}\right), \\
u_{2}\left(x_{0}+p\right)=u_{3}\left(x_{0}+p\right), u_{2}^{\prime}\left(x_{0}+p\right)=u_{3}^{\prime}\left(x_{0}+p\right), \\
u_{3}\left(x_{0}+1\right)=u_{4}\left(x_{0}+1\right), u_{3}^{\prime}\left(x_{0}+1\right)=u_{4}^{\prime}\left(x_{0}+1\right), \\
u_{4}\left(x_{0}+1+p\right)=u_{5}\left(x_{0}+1+p\right), u_{4}^{\prime}\left(x_{0}+1+p\right)=u_{5}^{\prime}\left(x_{0}+1+p\right), \\
u_{5}(\tilde{T})=1 .
\end{gathered}
$$

Here, as before, $p \equiv T-2 x_{0}-1(\bmod 1), P=\min \left\{n \in \mathbb{N}: n \geq T-2 x_{0}-1\right\}$, and

$$
g_{1}=\frac{G P}{P+1-p}, \quad g_{2}=\frac{G(P+1)}{P+1-p},
$$

by Theorem 6.6. Note that this is not exactly the same problem as (49-50), since the periodic section has been reduced from $P$ periods to a single period, and the solution is defined correspondingly on a smaller domain of length

$$
\tilde{T}=2 x_{0}+p+1 \text {. }
$$

This allows us to use the decomposition in five subdomains for any $T$, which facilitates computation. This is illustrated in Figure 12. 


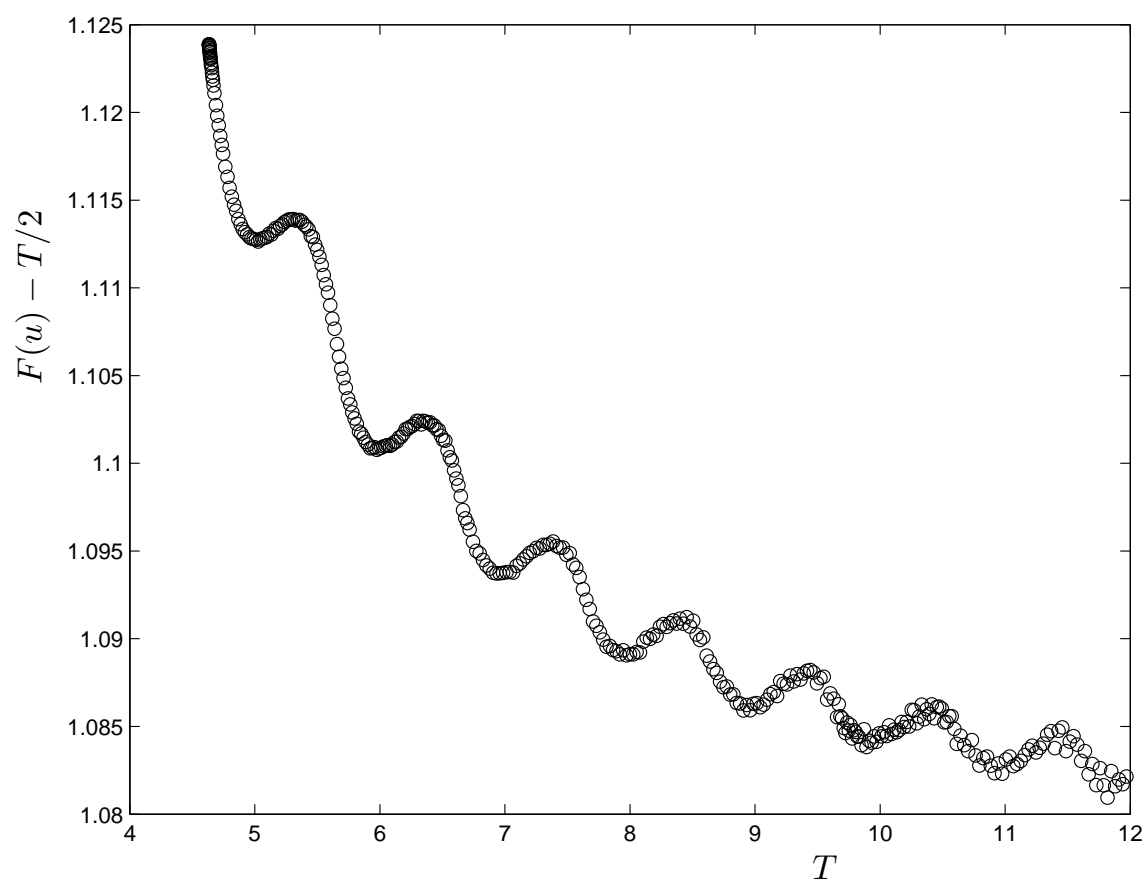

Figure 11. Behaviour of $F(u)-T / 2$ as a function of domain size $T$ for system (56)-(60) computed with AUTO. As $T$ grows, $F(u)-T / 2$ oscillates towards 1 , the energy of $u \equiv 0$ on a unit length interval.

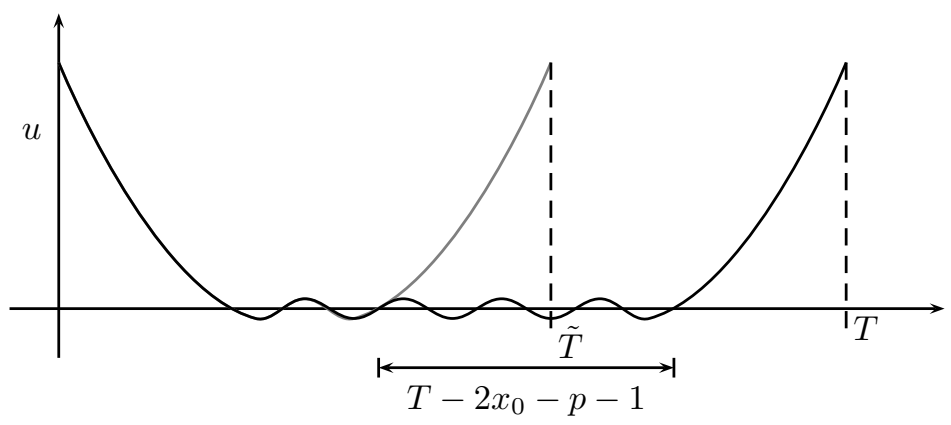

FiguRE 12. Schematic picture of the idea behind $\tilde{T}=2 x_{0}+p+1$. Since $u$ (solid black line) is periodic between $x_{0}$ and $T-x_{0}$, we can cut out an interval of length $T-2 x_{0}-p-1$ and find the corresponding solution on $[0, \tilde{T}]$.

We now vary $x_{0}$ and $G$ to find solutions of system (61)-(67) that satisfy

$$
u_{2}\left(x_{0}\right)=u_{3}\left(x_{0}+1\right), \quad \int_{x_{0}}^{x_{0}+p} u_{2}+\int_{x_{0}+p}^{x_{0}+1} u_{3}=0,
$$

using a standard Matlab boundary-value problem solver, bvp4c. An example solution is drawn in Figure 3 in which we have used $\alpha=1 / 2 \pi, r=1$. Note that 
all analysis in this paper assumes zero rod thickness; in Figure 3 the rod has been artificially fattened for better viewing.

\section{REFERENCES}

[1] S.S. Antman. Nonlinear problems in elasticity. Springer-Verlag, 1995.

[2] J.G. Blom and M.A. Peletier. A continuum model of lipid bilayers. Technical Report MASR0229, CWI, 2002.

[3] J. Cantarella, J.H.G. Fu, R. Kusner, J.M. Sullivan, and N.C. Wrinkle. Criticality for the Gehring link problem. arXiv: math.DG/0402212, 2004.

[4] J. Cantarella, R.B. Kusner, and J.M. Sullivan. On the minimum rope length of knots and links. Inventiones Mathematicae, 150:257-286, 2002.

[5] B.D. Coleman and D. Swigon. Theory of supercoiled elastic rings with self-contact and its application to DNA plasmids. J. Elasticity, 60:173-221, 2000.

[6] B.D. Coleman, D. Swigon, and I. Tobias. Elastic stability of DNA configurations II. Supercoiled plasmids with self-contact. Physical Review E, 61:759-770, 2000.

[7] E. Doedel, A. Champneys, T. Fairgrieve, Y. Kuznetsov, B. Sandstede, and X. Wang. Auto97: Continuation and bifurcation software for ordinary differential equations; available by ftp from ftp.cs.concordia.ca in directory pub/doedel/auto.

[8] W.B. Fraser and D.M. Stump. The equilibrium of the convergence point in two-strand yarn plying. Int. J. Solids Structures, 35(3-4):285-298, 1998.

[9] D. Gilbarg and N.S. Trudinger. Elliptic partial differential equations of second order. Springer-Verlag, 1977.

[10] O. Gonzalez, J.H. Maddocks, F. Schuricht, and H. von der Mosel. Global curvature and self-contact of nonlinearly elastic curves and rods. Calculus Variations, 14:29-68, 2002.

[11] G.H.M. van der Heijden. The static deformation of a twisted elastic rod constrained to lie on a cylinder. Proc. Roy. Soc. London A, 457:695-715, 2001.

[12] G.H.M. van der Heijden, S. Neukirch, V.G.A. Goss, and J.M.T. Thompson. Instability and self-contact phenomena in the writhing of clamped rods. Int. J. Mechanical Sciences, 45:161$196,2003$.

[13] G.H.M. van der Heijden, M.A. Peletier, and R. Planqué. A consistent treatment of link and writhe for open rods. arXiv: math-ph/0310057, 2003.

[14] G.H.M. van der Heijden and J.M.T. Thompson. Lock-on to tape-like behaviour in the torsional buckling of anisotropic rods. Physica D, 112:201-224, 1998.

[15] J.H. Maddocks. Stability and folds. Arch. Rat. Mech. Anal., 99:301-328, 1987.

[16] S. Neukirch and G.H.M. van der Heijden. Geometry and mechanics of uniform $n$-plies: from engineering ropes to biological filaments. J. Elasticity, 69:41-72, 2002.

[17] M.H. Protter and H.F. Weinberger. Maximum principles in differential equations. PrenticeHall, 1967.

[18] F. Schuricht and H. von der Mosel. Characterization of ideal knots. Calculus Variations, 19:281-315, 2004.

[19] F. Schuricht and H. von der Mosel. Euler-Lagrange equations for nonlinearly elastic rods with self-contact. Arch. Rat. Mech. Anal., 168:35-82, 2003.

[20] D.M. Stump and G.H.M. van der Heijden. Birdcaging and the collapse of rods and cables in fixed-grip compression. Int. J. Solids and Structures, 38:4265-4278, 2001.

[21] J.M.T. Thompson, G.H.M. van der Heijden, and S. Neukirch. Supercoiling of DNA plasmids: mechanics of the generalized ply. Proc. R. Soc. London, Series A, 458:950-985, 2002.

[22] I. Tobias, D. Swigon, and B.D. Coleman. Elastic stability of DNA configurations I. general theory. Physical Review E, 61:747-758, 2000. 
(†) Centre for Nonlinear Dynamics, University College London, Gower Street, LonDON WC1E 6BT, UK.

(†) Dept. of Mathematics and Computing Science, Technical University Eindhoven, PO Box 513, 5600 MB Eindhoven, The Netherlands.

(§) Centrum voor Wiskunde en Informatica, Kruislaan 413, 1098 SJ Amsterdam, The NETHERLANDS.

E-mail address: †UCESGVD@UCL.AC.UK, †MPELETIE@WIN.TUE.NL, §RPLANQUE@CWI.NL. 